\title{
Die Verfassung von Vertragsverbindungen
}

Wer sich mit Vertragsverbindungen auseinandersetzt, wird schnell feststellen, dass diesem Phänomen mit herkömmlicher Rechtsdogmatik nicht beizukommen ist. Der tiefere Grund dafür liegt in einem Emergenzphänomen: Vertragsverbindungen lassen aus bilateralen Rechtsbeziehungen - aus zweiseitigen schuldrechtlichen Verträgen neue Erwartungsordnungen entstehen, in die mehrere, zuweilen zahlreiche selektiv miteinander interagierende Akteure eingebunden sind (man denke z.B. an Franchiseoder Interbankzahlungssysteme). Für diese neuen Ordnungen ist das traditionelle Vertragsrecht blind. Eine angemessene Reaktion besteht darin - so meine These -, diese emergenten Erwartungsordnungen einer Rechtsverfassung höherer Ordnung zuzuführen. Mit »Rechtsverfassung höherer Ordnung « ist gemeint, dass aus den obligationenrechtlichen Regeln heraus, welche für die einzelnen Kontrakte gelten, die die Vertragsverbindung konstituieren, eine neue (also ebenfalls emergente) Rechtsordnung entwickelt wird. Gelingen kann das Design einer solchen »Verbund-Verfassung « nur, soweit rechtssoziologische Erkenntnisse in die zivilistische Dogmatik einfließen - und zwar gerade deshalb, weil man es bei Vertragsverbindungen nicht durchwegs mit rechtlichen, aber eben doch mit zuweilen juristisch relevanten Normativitäten zu tun hat. Nachstehend wird zunächst besprochen, wie das moderne Vertragsrecht die Blende in diesem Sinne öffnen kann (I.). Darauf aufbauend wird spezifisch den Vertragsverbindungen und ihren Eigenarten im Spiegel der jüngeren schweizerischen Rechtsprechung nachgegangen (II.). In einem abschließenden Teil wird gefragt, wie auf die Erwartungsordnungen, die aus der Verbindung von Verträgen emergieren, privatrechtsdogmatisch reagiert werden kann, insbesondere wie eine schuldrechtliche Verfassung von Vertragsverbindungen de lege lata zu entwerfen ist (III.).

$I$.

Wie fließen dem Recht die Informationen über seine Umwelt zu, die es benötigt, um die Gesellschaft zu verrechtlichen? Unter den Bedingungen einer funktionalen Ausdifferenzierung der Gesellschaft ist das eine Frage der Reflexion des Rechtssystems: Durch Reflexion lernt das Recht sich als Teil seiner Umwelt zu verstehen, was es zugleich zwingt - und das ist hier der springende Punkt -, die seine Aufgabe ermöglichenden und unterstützenden Vorstellungen über seine Umwelt - also: Wirklichkeitskonstruktionen - zu entwickeln ${ }^{1}$. Reflexion setzt innerhalb eines jeden Sozialsystems die Schaffung eines »Reflexionsapparates« voraus, was im Rechtssystem durch die Erarbeitung einer Rechtstheorie (im Singular) geschieht ${ }^{2}$. LUHMANN hat darauf hinge-

1 Vgl. dazu aufschlussreich Marco Wehr (2000) »Die Inseln der Propheten: Emergenz kognitiver Repräsentationen«, in: Thomas Wägenbaur (Hg.) Blinde Emergenz? Interdisziplinäre Beiträge zu Fragen kultureller Evolution, Heidelberg: Synchron, 65-74.

2 Vgl. zum Ganzen Marc Amstutz (2002) »Historizismus im Wirtschaftsrecht: Überlegungen zu einer evolutorischen Rechtsmethodik «, in: Rainer Schweizer, Herbert Burkert und Urs Gasser (Hg.) Festschrift für Jean Nicolas Druey zum 65. Geburtstag, Zürich: Schulthess, 21 m.w.N. 
wiesen, dass man sich »die Abstraktionsebene von ,Theorie' als einen Mechanismus der strukturellen Kopplung vorstellen [kann], der die Reflexionsleistungen des Rechtssystems ... mit den Theorieleistungen des Wissenschaftssystems verbindet $\aleph^{3}$. Hier nun liegen die Chancen - aber auch die Risiken - eines Zugangs des Rechts zu den »mondes intérieurs « der Gesellschaft: die Chancen, weil so das Recht Beobachtungs-Instrumente zur Verfügung bekommt, um die Verhältnisse und die Prozesse in seiner Umwelt besser erfassen $\mathrm{zu}$ können. Diese Instrumente sind die von FouCAULT beschriebenen »Epistemen « ${ }^{4}$, die der Rechtstheorie (wie den Reflexionstheorien anderer Sozialsysteme) erlauben, die Wirklichkeit in einem bestimmten Sinne zu deuten. Aber eben - und das sind die Risiken - nur in einem bestimmten Sinne (eine »Gesamtsicht « ist nicht zu haben). Weil »Epistemen« im Wissenschaftsbetrieb in Überfülle angeboten werden, muss das Recht eine durch nichts gesicherte Selektion treffen - und das Risiko eingehen, die Gesellschaft zu verpassen. Aus diesem Selektionszwang erklärt sich die grassierende Zahl (»Variation«) von konkurrierenden Rechtstheorien (diesmal im Plural), die alle für sich beanspruchen, die Rechtstheorie (als Reflexionsapparat des Rechts) zu verkörpern: Naturrechtstheorien unterschiedlichster Prägung, Positivismen aller Art, Law \& Economics zahlreicher Färbungen, Diskurs- und Argumentationslehren, Feminismen, Critical Theories usw. (ad aeternum).

Ein Weg, um das Dilemma dieser im Grunde unmöglichen, aber dennoch notwendigen Selektion zu umgehen, könnte darin liegen, dass das Recht in seiner Selbstbeschreibung und seinen Operationen auf die Kausalitäts- oder Teleologiemuster, die den eben erwähnten Rechtstheorien mehr oder weniger ausgeprägt innewohnen, verzichtet und konsequent auf eine »Evolutions-Semantik « ${ }^{5}$ umpolt. VON FOERSTER hat mit vorbildlicher Nüchternheit angegeben, worin die Stärken einer solchen Semantik zu erblicken sind: »Ontologisch Unerklärbares kann sich als ontogenetische Notwendigkeit erweisen. Der Nabel ist ein ontologischer Witz, ein Schnörkel, ein barockes Rätsel auf dem eigenen Bauch. Ontogenetisch würden wir jedoch ohne ihn nicht sein. Evolutionisten und Kreationisten suchen gleichermassen eine ontogenetische Erklärung für ein andernfalls unerklärbares Phänomen: Wir sind da! « ${ }^{6}$. Hierin liegt wohl der Grund dafür, dass neuerdings in den Sozialwissenschaften eine Renaissance der Evolutionstheorie im Gange ist, die zuweilen als ein »interdisciplinary paradigm shift« gedeutet wird ${ }^{7}$.

Ist man, wie hier, auf der Suche nach der Möglichkeit einer »realistischeren« Annäherung des Rechts an die »mondes intérieurs« der Gesellschaft, mag dieses epistemo-

3 Niklas Luhmann (1993) Das Recht der Gesellschaft, Frankfurt a.M.: Suhrkamp, 564.

4 Michel Foucault (1974) Die Ordnung der Dinge: Eine Archäologie der Humanwissenschaften, Frankfurt a.M.: Suhrkamp.

5 Markus Heilmann (2000) »Angepasste Selbstbeschreibung: Zu Niklas Luhmanns ,Evolution der Evolution' ", in: Thomas Wägenbaur (Hg.) Blinde Emergenz? Interdisziplinäre Beiträge zu Fragen kultureller Evolution, Heidelberg: Synchron, 145.

6 Heinz von Foerster (1993) Wissen und Gewissen: Versuch einer Brücke, Frankfurt a.M.: Suhrkamp, 370.

7 David Shaw (2002) »An Interdisciplinary Paradigm Shift«, in: Philip Pomper und David Shaw (Hg.) The Return of Science: Evolution, History, and Theory, Lanham: Rowman \& Littlefield, $1 \mathrm{ff}$. 
logische Manöver aufs Erste überraschen, weil damit im Gegensatz zu den gängigen Epistemologien eine Rücknahme des Anspruchs auf »Erklärung der Welt« verbunden ist. Aber aus einem solchen frei gewählten Verzicht können sich, wie ich meine, auch neue Chancen ergeben. Welche Art von heuristischer Rücknahme hier genau gemeint ist, lässt sich wohl am besten wissenschaftshistorisch darlegen: Lange haben sich die Sozialwissenschaften am »mechanistischen « (deterministischen) Ideal der Naturwissenschaften, das namentlich mit cartesianischen und newtonschen Rationalitätsvorstellungen verbunden ist, orientiert ${ }^{8}$. Im Mittelpunkt stand die Annahme, es gäbe in sämtlichen Lebensbereichen so etwas wie unveränderliche Wahrheiten, und darin erblickte man die Möglichkeit für eine Prognosefähigkeit gesellschaftlicher (geschichtlicher, wirtschaftlicher, soziologischer, juridischer usw.) Prozesse. Diese Träume haben sich allmählich zerschlagen, dies wegen vielfältigster Erfahrungen, wie die logischen Entdeckungen GöDELs ${ }^{9}$, die rekurrierenden und nicht auszumerzenden Paradoxien in Wissenschaft und Leben ${ }^{10}$, die Realisierung der fehlenden Allgemeingültigkeit physischer Gesetze ${ }^{11}$ usw. Allmählich schlich sich Unbestimmtheit in die Wirklichkeitsbildung und die Wirklichkeitsbilder der Wissenschaft ein, aber dies in ganz spezifischer Form: als »evolutionary uncertainty" - evolutionäre Unsicherheit -, wie dies MCNEILL trefflich genannt hat ${ }^{12}$. Erkannt wurde, dass weder die Elemente eines Wissenssystems noch die daraus abgeleiteten Gesetzlichkeiten zahlenmäßig beschränkt, sondern re vera unbeschränkt sind ${ }^{13}$. Die Möglichkeit generalisierter Erklärungen schwand dahin und man realisierte, dass alles, was "gewusst « werden kann, es eben nur situativ, punktuell, kurzum: zeit- und kontextbezogen, sein kann.

Für die Sozialwissenschaften im allgemeinen und für die Rechtswissenschaft im besonderen bedeutet diese »Entdeckung « etwas ganz Spezifisches, das vielleicht MELEGHY in jüngerer Zeit am präzisesten ausgedrückt hat. Dieser weist darauf hin, dass die Evolutionstheorie kein wie auch immer geartetes universelles Gesetz enthält. Hingegen gibt sie einen Mechanismus (oder Algorithmus) an, der »es grundsätzlich vermag, die zielgerichteten rationalen Handlungen eines Menschen genauso wie die zielgerichtete planvolle Tätigkeit eines Schöpfers nachzuahmen $\aleph^{14}$. Was also im 19. Jahrhundert als der große Nachteil der

8 Die wohl beeindruckendste Analyse dieser Bezüge (am Beispiel des Verhältnisses Ökonomie / Physik) liefert Nicholas Georgescu-Roegen (1971) The Entropy Law and the Economic Process, Cambridge, Mass.: Harvard University Press.

9 Vgl. etwa Niklas Luhmann (1990) Die Wissenschaft der Gesellschaft, Frankfurt a.M.: Suhrkamp, $506 \mathrm{ff}$.

10 Grundlegend Willard Quine (1976) The Ways of Paradox, Cambridge, Mass.: Harvard University Press.

11 Vgl. etwa Henri Atlan (1999) La fin du »tout génétique«? Vers de nouveau paradigmes en biologie, Paris: INRA, $11 \mathrm{ff}$.

12 McNeill (Fn. 15) 23.

13 Vgl. etwa Ilya Prigogine (1994) Les lois du chaos, Paris: Flammarion, 16 ff.

14 Tamás Meleghy (2003) »Methodologische Grundlagen einer evolutionären Soziologie«, in: ders. und Heinz Niedenzu (Hg.) Soziale Evolution: Die Evolutionstheorie und die Sozialwissenschaften, Wiesbaden: Westdeutscher Verlag, 114-162, 132 f.: »Darwin zeigte uns, dass der Mechanismus der natürlichen Auslese grundsätzlich die zweck- und planvolle Tätigkeit eines Schöpfers nachahmen kann, ebenso auch vernünftige, zweck- oder zielgerichtete menschliche Handlungen«. 
Evolutionstheorie galt ${ }^{15}$, nämlich ihre Unfähigkeit zur Prognosestellung und ihre Beschränkung auf die Beschreibung kontingenter Veränderungsprozesse ${ }^{16}$, ist unter diesen Umständen vielleicht ihre heimliche Stärke. Denn Erkenntnis kann dann auf die Prämisse eines universellen Gesetzes (das sich gerade im Sozialen niemals finden lässt) verzichten: »In den Trümmern des alten Essenzkosmos mit seiner Einteilung in Gattungen und Arten war die Darwinsche Evolutionstheorie entstanden, die zeigen konnte, dass man auf einen Schöpfungsplan verzichten und trotzdem erklären kann, dass in der Welt der Lebewesen auf Grund von Selektionsvorgängen nichtbeliebige Verhältnisse herrschen ${ }^{17}$.

Für das Recht lägen die Chancen einer evolutionstheoretischen Epistemologie darin, das Schockerlebnis seiner Entzauberung im 19. und 20. Jahrhundert, die in der Einsicht bestand, dass Recht weder im Himmel noch auf Erden an etwas (»Gott«, Vernunft«, »Gerechtigkeit«) festgemacht ist, endlich zu verarbeiten: Trotz der Unmöglichkeit einer »Planung der Gesellschaft« durch das Recht - eine Unmöglichkeit, die nur gerade noch vom Gesetzespositivismus bestritten wird, der sich zur Übertünchung dieser Tatsache allerlei Fiktionen (wie z.B. der »Gesetzgeber «, der »gesetzgeberische Wille« oder der »Gesetzeszweck «) bedient - könnte über diesen Weg eine alte Schuld der Rechtswissenschaft beglichen werden: der Nachweis, dass Recht in seinem Zugriff auf die Gesellschaft nicht willkürlich vorgehen kann, sondern an gewisse Gesetzesmässigkeiten (oder: »Bedingungen «) gebunden ist - und sei dies auch nur in der Form, dass zwischen Recht und Gesellschaft ein evolutorisches Gleichgewicht, eine »reziprok produzierte Homöostase « bestehen muss.

Aber wie kann das Recht seine lebensweltlichen Gegenstände im geschilderten Sinne »evolutionstheoretisch « beobachten?

\section{II.}

Letztlich ist das eine Frage der Wahl eines der zahlreichen Theoriedesigns, die in der Evolutionstheorie heute offeriert werden ${ }^{18}$. In den Sozialwissenschaften scheint sich seit einiger Zeit das darwinistische (systemtheorisch modifizierte) Muster der Trias Variation / Selektion / Retention durchzusetzen ${ }^{19}$. Verfährt man nach diesem Muster,

15 Vgl. William McNeill (2002) »History and the Scientific Worldview«, in: Philip Pomper und David Shaw (Hg.) The Return of Science: Evolution, History, and Theory, Lanham: Rowman \& Littlefield, 13-15; vgl. mit umfassenden Ausführungen zum erkenntnistheoretischen Status der Evolutionstheorie Bernard Feltz (1999) »Auto-organisation, sélection et émergence dans les théories de l'évolution«, in: Bernard Feltz, Marc Crommelinck und Philippe Goujon (Hg.) Auto-organisation et émergence dans les sciences de la vie, Bruxells: Ousia, 475-501, 483 ff. m.w.N.

16 Vgl. Heilmann (Fn. 5) 143 f.

17 Niklas Luhmann (2000) Die Politik der Gesellschaft, Frankfurt a.M.: Suhrkamp, 408.

18 Eine Übersicht aus sozialwissenschaftlicher Sicht geben Tamás Meleghy und Heinz Niedenzu (2003) »Einleitung: Die Evolutionstheorie und die Sozialwissenschaften«, in: dies. (Hg.) Soziale Evolution: Die Evolutionstheorie und die Sozialwissenschaften, Wiesbaden: Westdeutscher Verlag, 9-32, $11 \mathrm{ff}$.

19 Vgl. Zu diesem Modell etwa Marcelo Neves (2000) Zwischen Themis und Leviathan: Eine schwierige Beziehung. Eine Rekonstruktion des demokratischen Rechtsstaates in Auseinandersetzung mit Luhmann und Habermas, Baden-Baden: Nomos, 13 ff. 
besteht die Herausforderung darin, in der Gesellschaft selber die Mechanismen der Produktion neuer sozialer Formen, der Auslese zwischen diesen Form-Angeboten und ihrer Setzung auf Dauer in der sozialen Praxis zu verorten ${ }^{20}$. Dann gewinnt man ein Verständnis für die Nichtbeliebigkeit von Gesellschaft, ohne dass dessen Formen mit ontologischen Notwendigkeiten oder absichtlichem Planen einer allwissenden Instanz in Verbindung gebracht werden müssen. Und vor allem - das ist vorliegend im Hinblick auf das Zusammenwirken von Recht und Gesellschaft zentral - gewinnt man so ein Verständnis von sozialen Phänomenen, das nicht davon abhängig ist, dass ein universelles Gesetz die Funktionsweise dieser Phänomene bestimmt.

In dieser Zielrichtung der Erklärung verdient der Ansatz Zustimmung, nicht jedoch im Beizug des geschilderten dreigliedrigen Analyserasters. Denn dieser greift insofern zu kurz, als er kein theoriegeleitetes Kriterium angibt, um zwischen mehreren möglichen evolutionstheoretischen Erklärungen des Lebendigen oder des Sozialen auszuwählen ${ }^{21}$. Ein solches Kriterium ist aber unerlässlich, weil eine mit Hilfe der Dreiheit Variation / Selektion / Retention vorgehende Analyse regelmäßig mehrere plausible evolutionstheoretische Hypothesen zulässt ${ }^{22}$. Deshalb wird man nach einem umfassenderen Analyseraster suchen müssen, der, wie die erwähnten Autoren sagen, die Bestimmung von »criteria to identify proper explanations among the substantial set of plausible pathways to any modern result« erlaubt $^{23}$. In seinem opus magnum The Structure Of Evolutionary Theory ${ }^{24}$ hat GoulD ein Analyseraster entwickelt, der die Angabe solcher Kriterien erlaubt und auch für die Sozialwissenschaften (insbesondere für die Rechtswissenschaft) produktiv sein könnte. GoulDs Modell baut auf drei Fragen - gekennzeichnet als »Agency«, »Efficacy « und »Scope ${ }^{25}$ - auf, von deren Beantwortung er die Schlüssigkeit der evolutionstheoretischen Erkärung abhängig macht:

(1) Agency: Wo befindet sich der »Ort«, wo die evolutorischen Kräfte ihre Wirkung entfalten? Die Evolution braucht einen $»$ locus of action $\aleph^{26}$ bzw. eine $»$ Selektionseinheit«. In der Biologie ging Darwin konsequent davon aus, dieser »Ort« sei der (individuelle) Organismus, bis man zu entdecken begann, dass weitere »units of selection« auf tieferen oder höheren Stufen (Gene, Zellen, Organe bzw. Gruppen, Arten usw.) gleichzeitig existieren, dass es also nebst mikroevolutorischen auch makroevolutori-

20 Beispielhaft durchgeführt für das Rechtssystem in der Studie von Marie Fögen (2002) Römische Rechtsgeschichten: Über Ursprung und Evolution eines sozialen Systems, Göttingen: Vandenhoeck \& Ruprecht.

21 Vgl. dazu eingehend Marc Amstutz (2002) »Rechtsgeschichte als Evolutionstheorie: Anmerkungen zum Theorierahmen von Marie Theres Fögens Forschungsprogramm«, 1 Rechtsgeschichte, 26-31.

22 Stephen Gould und Richard Lewontin (1979) «The spandrels of San Marco and the Panglossian paradigm: a critique of the adaptionist programme", B 205 Proceedings of the Royal Society, 581-598, 588.

23 Gould und Lewontin (Fn. 22) 588.

24 Stephen Gould (2002) The Structure Of Evolutionary Theory, Cambridge, Mass.: Belknap.

25 Dieses Schema prägt den Aufbau des ganzen Buches; kurz erläutert ist es an folgender Stelle: Gould (Fn. 24) $14 \mathrm{f}$.

26 Gould (Fn. 24) 14. 
sche Phänomene gibt ${ }^{27}$. Von dieser sog. hierarchischen Theorie der Selektion sollten sich die Sozialwissenschaften und zumal die Rechtswissenschaft inspirieren lassen: Auch im Bereich der gesellschaftlichen Evolution muss eine Beschränkung der Optik auf eine einzige Wirkungsebene der evolutorischen Kräfte, wie z.B. die Ebene der »Kommunikation« oder des »Sozialsystems«, vermieden werden.

(2) Efficacy: Braucht es neben dem Mechanismus der natürlichen Auslese nicht zusätzliche Erklärungselemente, um den evolutorischen Prozess zu verstehen? Der klassische Darwinismus verneint diese Frage mit dem Argument, dass bei ausreichender Variation eine langsame und allmähliche (gradualistische) Beseitigung von individuellen Organismen, die an ihre Umwelt nicht angepasst sind, genügt, um die Erzeugung neuer, überlebenstauglicher Lebensformen sicherzustellen ${ }^{28}$. Demgegenüber hebt die moderne Evolutionsbiologie die Bedeutung von sog. »constraints « hervor: Zwar sei die »externe« Perspektive des Darwinismus durchaus zutreffend, aber insofern unvollständig, als Organismen »innere « Entwicklungsschranken besitzen, die im evolutorischen Prozess eine wichtige Rolle spielen (krass gesagt: aus dem Elefanten werden auch Jahrmillionen Variation und Selektion keine Katze machen; sein Bauplan schließt das aus $)^{29}$. Nun gibt es solche $»$ constraints « offenbar auch in der sozialen Evolution: Historische oder strukturelle Gründe kanalisieren die in der Gesellschaft wirkenden selektiven Kräfte gelegentlich in eine unerwartete Richtung ${ }^{30}$.

(3) Scope: Kann die Diversität der Welt allein mit den mikroevolutorischen Mechanismen des Darwinismus erklärt werden? Bedarf es hierfür nicht der zusätzlichen Berücksichtigung »nichtevolutorischer« (d.h. nicht algorithmischer) Ereignisse (wie z.B. Klimaveränderungen oder großflächige Meteoriteneinschläge)? Angesprochen ist die Kontroverse zwischen Uniformitaristen und Katastrophisten: Während jene einem strikten Darwinismus huldigen und die natürliche Auslese als exklusive Ursache ökologischer Vielfalt ansehen, vertreten diese die Auffassung, diese Vielfalt könne nur unter Mitberücksichtigung anderer causae erklärt werden ${ }^{31}$. In der gegenwärtigen Evolutionsbiologie gilt der Gesichtspunkt des Katastrophismus als anerkannt und ihm sollte sich auch eine Theorie sozialer Evolution öffnen: Gerade weil Sozialsysteme strukturell gekoppelt sind, d.h. sich gegenseitig auf ihre Strukturen verlassen (z.B. verlässt sich die Wirtschaft darauf, dass es den juristischen Vertrag gibt, oder die Politik

27 Die Annahme einer Mehrheit und Hierarchie der Selektionsebenen wird vor allem damit begründet, dass es Selektionen gibt, die auf der klassisch-darwinistischen Ebene der Organismen unsichtbar bleiben; vgl. David Depew und Bruce Weber (1996) Darwinism Evolving: Systems Dynamics and the Genealogy of Natural Selection, Cambridge, Mass.: MIT, $381 \mathrm{ff}$.

28 Vgl. in erster Linie Stephen Gould (1982) »Darwinism and the Expansion of Evolutionary Theory «, 216 Sci., 380-387, 381.

29 Vgl. dazu eingehend Marc Amstutz (2001) Evolutorisches Wirtschaftsrecht: Vorstudien zum Recht und seiner Methode in den Diskurskollisionen der Marktgesellschaft, BadenBaden: Nomos, 266 ff. m.w.N.

30 Vgl. z.B. Viktor Vanberg (1996) »Institutional Evolution Within Constraints«, 152 JITE, 690-696.

31 Vgl. für einen einleuchtenden historischen Abriss der Uniformitarismus / KatastrophismusKontroverse Depew und Weber (Fn. 27) 95 ff. 
darauf, dass der Staat von einem Grundgesetz verfasst ist), können »jumps « in einem bestimmten System (z.B. die Erfindung der Schrift, des Buchdruckes oder des Internets) für andere Systeme massive evolutorische Konsequenzen haben.

Ich möchte dieses Muster evolutorischer Analyse vorliegend in der Weise einsetzen, dass es - in Beibehaltung der vorne eingenommenen Doppelperspektive von einem »Außen« und einem »Innen« des Rechts - zunächst der Erforschung der in Wirtschaft und Gesellschaft anzutreffenden Vernetzungsphänomene (oder Netzwerkphänomene) dient. Diese Phänomene (soviel darf unterstellt werden) hängen aufs Engste mit dem Auftreten von Vertragsverbindungen im Recht zusammen ${ }^{32}$. In einem zweiten Schritt ist dann zu untersuchen, wie die internen Strukturen des Rechts auf diese Phänomene reagieren (sollten). Zu diesem Zweck beginne ich mit einer Beobachtung der sozialen Praxen der Vertragsverbindung im Spiegel der neueren Rechtsprechung ${ }^{33}$ :

Als erstes ist auf eine Reihe von Urteilen einzugehen, die anschaulich machen, dass im institutionellen Bereich Variationen nicht bloß auf einer einzigen Ebene produziert werden. Wie in der Natur sich das Geschehen nicht ausschließlich auf der Ebene des Organismus abspielt, so können im Sozialen privatautonome Ordnungen (Verträge, Gesellschaften usw.) auf höheren Ebenen Wirkungen haben, die emergente Ordnungen erzeugen und die unsichtbar bleiben, wenn die GoulDsche »Agency« nicht thematisiert wird. Geradezu beispielhaft kommt dieser Aspekt in der Umschreibung des Netzwerkes von Ober- und Untermietverhältnis zum Ausdruck, die das Bundesgericht in BGE 120 II 112 vorgenommen hat. Es lohnt sich, die einschlägige Passage im Volltext wiederzugeben:

»Die Untermiete ist durch zwei hintereinandergeschaltete Mietverträge über dieselbe Sache gekennzeichnet. Der Grundsatz der Relativität der Schuldverhältnisse lässt dabei zwar die rechtliche Selbständigkeit der beiden Vertragsverhältnisse unschwer erkennen, hilft aber nicht darüber hinweg, dass sie desungeachtet untereinander verbunden sind ..., namentlich die Hauptmiete die Rechtsmacht des Untervermieters beschränkt ... und der daraus folgende wirtschaftliche Verbund der beiden oder mehreren Verträge auch rechtliche Lösungen erheischt, welche den nicht mehr nur zwei Personen betreffenden Interessenkonflikten gerecht zu werden vermögen ... . Z Zu Recht wird daher darauf hingewiesen, dass zwischen Hauptvermieter und Untermieter ein rechtliches Sonderverhältnis, wenn auch kein unmittelbares Vertragsverhältnis, be-

32 Vorliegend wird auf den Zusammenhang von Netzwerkphänomenen auf sozialer Ebene und Vertragsverbindungen auf juristischer Ebene nicht ausdrücklich eingegangen; vielmehr wird auf der Grundlage von Teubners Arbeiten angenommen, dass beide Erscheinungen »zwangsgekoppelt « sind; vgl. Gunther Teubner (2004) Netzwerk als Vertragsverbund: Virtuelle Unternehmen, Franchising, Just-in-Time in sozialwissenschaftlicher und juristischer Sicht, Baden-Baden: Nomos.

33 Mit dem Fokus auf die jüngere schweizerische Rechtsprechung wird lediglich eine umfangmässige Beschränkung des Analysematerials bezweckt; keineswegs soll gesagt sein, die ältere Rechtsprechung sei im Kontext belanglos. Als mitlaufende Referenzen zu betrachten sind deshalb: BGE 25 II 473; 63 II 414; 94 II 161; 94 II 355; 97 II 390; AGVE 1981, 38 ff.; LGVE 1986 I, Nr. 35, 57 ff.; ZGGVP 1991/92, 163 ff.; PKG 1991, Nr. 61, 203 ff.; PKG 1992, Nr. 63, 230 ff.; PKG 1994, Nr. 50, 160 ff.; vgl. daneben auch BGE 116 II 634; BGE vom 26. September 2001, Nr. 4C.161/2001; BGE vom 9. Juli 2003, Nr. 4C.163/2002. 
steht ... . Dabei ist ebenfalls zu beachten, dass der Vermieter nicht mehr frei ist, Untermiete zu gestatten oder nicht (Art. 262 Abs. 2 OR); er mithin von Gesetzes wegen verpflichtet ist, in bestimmt geartete Beziehungen zu einem Drittbenützer zu treten ${ }^{34}$.

Erkennbar wird in diesen Ausführungen, dass der mietgeschäftliche Verbund zwar ausschließlich aus bipolaren Rechtsbeziehungen besteht, dass aber diese Beziehungen funktional zusammengehören und sich in dieser Zusammengehörigkeit im Rechtsverkehr »bewähren « müssen ${ }^{35}$. Evolutionstheoretisch ließe sich dieser Befund so ausdrücken, dass nicht bloß die primäre Ebene der Einzelvereinbarungen, sondern auch die sekundäre Ebene der Ordnung, die aus der Koppelung der Vereinbarungen fließt, selektiven Umweltkräften ausgesetzt ist.

Diese sekundäre Ordnungebene kommt auch in BGE 115 II 452 zum Vorschein, in dem es um zwei Verträge zwischen der Betriebsgesellschaft der Klinik Y. und Dr. med. X. ging. Die Parteien hatten am 6. Mai 1983 einen sog. Zusammenarbeitsvertrag abgeschlossen, der die Tätigkeit von X. als Belegsarzt der Klinik regelte. Die Vereinbarung wurde auf unbestimmte Zeit mit einer sechsmonatigen Kündigungsfrist eingegangen (Art. 5 des Vertrages). Knapp ein Jahr später, nämlich am 1. April 1984, kam es zwischen denselben Parteien zum Abschluss eines Mietvertrages, der die Überlassung von Räumlichkeiten im Klinikgebäude zwecks Betriebs einer privaten Arztpraxis durch X. zum Gegenstand hatte. Auch dieser Vertrag war zeitlich unbefristet. Seine Beendigungsordnung wurde durch Verweisung auf die entsprechende Regelung im Zusammenarbeitsvertrag festgelegt, indem als Kündigungsfrist »der im Art. 5 des Zusammenarbeitsvertrages vom 06.05 .83 vereinbarte Zeitraum« gelten sollte. Am 3. Januar 1989 kündigte die Klinik Y. AG den Zusammenarbeitsvertrag und forderte X. auf, die Räume seiner Arztpraxis in der Klinik bis zum 7. Juli 1989 freizugeben. Daraufhin ersuchte X., gestützt auf Art. 267a altOR (Art. 272 OR) um Erstreckung des Mietverhältnisses bis Ende 1990, was ihm sowohl vor erster ${ }^{36}$ als auch zweiter Instanz verweigert wurde. Das Bundesgericht folgte den Vorinstanzen im Ergebnis, aber nicht in der Begründung:

Die Vorinstanzen haben nicht zwei separate Verträge, sondern eine einzige (gemischte) Vereinbarung unterstellt, was ihnen erlaubte, (wohl in Befolgung der Absorptionstheorie ${ }^{37}$ ) die Anwendung von Art. 267a altOR (Art. 272 OR) mit dem Argument $\mathrm{zu}$ umgehen, dass die kooperationsrechtlichen Elemente der Vereinbarung

34 BGE 120 II 115 (H.v.Verf.).

35 Die im Schrifttum vorherrschende Vision, dass im rechtsgeschäftlichen Verbund der Grundsatz der gegenseitigen Unabhängigkeit der Verträge gelte (vgl. etwa Romeo Cerutti (1990) Der Untervertrag, Freiburg i.Ue.: Universitätsverlag, 54 ff. N 214 ff.), bestreitet im Ergebnis eine solche Mehrebenen-Betrachtung.

36 Der Sachverhalt spricht in diesem Zusammenhang davon, dass die erste Instanz »dem Grundsatz nach einen Anspruch des Klägers aus Art. 267a OR [verneinte], ... das Mietverhältnis aber dennoch bis zum 31. Oktober 1989 [erstreckte] « (BGE 115 II 453). Nähere Angaben über die Begründung dieser Entscheidung fehlen.

37 Vgl. zur Absorptionstheorie Walter Schluep und Marc Amstutz (2003)»Vor Art. 184 ff.«, in: Heinrich Honsell, Nedim Vogt und Wolfgang Wiegand (Hg.) Basler Kommentar zum schweizerischen Privatrecht: Obligationenrecht I, 3. Aufl., Basel: Helbing und Lichtenhahn, $878 \mathrm{~N} 13$. 
dominant seien und die für die »beigemischten« (mietrechtlichen) Elemente vorgesehenen Rechtsfolgen »aufsaugen « und unanwendbar machen würden ${ }^{38}$. Demgegenüber ist das Bundesgericht »von einer Mehrheit von Verträgen«, nämlich von zwei eigenständigen Vereinbarungen ausgegangen, dies in der Überlegung, dass zwischen den Parteien kein einheitlicher Akt der Konsensbildung vorlag ${ }^{39}$. Im Anschluss an die Feststellung, dass »die Frage, ob auch Benützungsverhältnisse einer Erstreckung zugänglich sind, die nicht auf einem reinen Mietvertrag beruhen, sondern ... auf einem Vertragskomplex, in welchem ein Mietvertrag mit anderen Verträgen verbunden ist«, $»[v]$ om Gesetzgeber nicht allgemein beantwortet wird $\aleph^{40}$, sucht es nach einer Antwort in der Lehre. Vergeblich, da diese sich nur zum gemischten Vertrag äußert und seine Erstreckbarkeit »einerseits von seinem Regelungsschwerpunkt ..., andererseits von einer Interessenabwägung im Einzelfall abhängig « macht $^{41}$. An diesem Punkt angelangt nehmen die Urteilserwägungen eine spektakuläre Wende. Ohne eigentliche Begründung wird behauptet, dass für den zusammengesetzten Vertrag »Entsprechendes ... gelten [muss] «. Wörtlich heißt es: »Auch hier ist die Erstreckung des Mietverhältnisses ausgeschlossen, wenn der damit gekoppelte Vertrag die Rechtsbeziehungen der Parteien schwergewichtig prägt und die Überlassung des Mietobjekts bloß als untergeordnete Nebenabrede erscheint. In jedem Einzelfall ist daher zu prüfen, welche Bedeutung die Parteien den gekoppelten Einzelverträgen im Hinblick auf die Gestaltung der Gesamtrechtslage beigemessen haben und in welchem Abhängigkeitsverhältnis diese Verträge nach ihrer rechtlichen und wirtschaftlichen Bedeutung zueinander stehen $\ldots \ll^{42}$.

Auch hier erfolgt der Zugriff des Rechts - als selektive Kraft in der Umwelt des autonomen Kooperationssystems, das X. und die Klinik Y. AG anhand von zwei schuldrechtlichen Verträgen in Platz gesetzt haben - auf Ebene der zwischen den Parteien geltenden Gesamtordnung: Nicht jeder Vertrag wird für sich allein betrachtet, sondern die Vertragsverbindung als solche rückt in den Mittelpunkt. Und dies, obschon das positive Recht eigentlich zu einer »einzelvertraglichen« Sicht der Dinge drängt. Denn Art. 267a altOR (Art. 272 OR) stellt zwingendes Recht dar ${ }^{43}$, das anwendbar wird, sobald im Sachverhalt die Tatbestandsmerkmale einer Miete von Wohn- oder Geschäftsräumen erfüllt sind. Da diese Merkmale unzweifelhaft vorhanden waren, hätte das positive Recht an sich nahe gelegt, den Mietvertrag vom 1. April 1984 einer partikularen, isolierten Betrachtung zu unterziehen. Das Bundesgericht hat

38 BGE 115 II 453.

39 Ausdrücklich wird das in den Erwägungen nirgends gesagt. Bei der Erläuterung der Eigenarten eines zusammengesetzten Vertrages erwähnt das Gericht aber, dass ein solcher u.a. »mangels eines einheitlichen Aktes der Konsensbildung « angenommen werden müsse (BGE 115 II 454).

40 BGE 115 II 454.

41 BGE 115 II 454.

42 BGE 115 II 454 (H.v.Verf.).

43 Vgl. statt anderer Peter Higi (1994)»Art. 253-265 OR«, in: Peter Gauch (Hg.) Kommentar zum Schweizerischen Zivilgesetzbuch: Obligationenrecht, Teilbd. V/2b: Die Miete, Zürich: Schulthess, 167 N 12 ff. 
indes die Einschlägigkeit von Art. 267a altOR (Art. 272 OR) schon gar nicht erst geprüft und mit seinem Fokus auf die »Gesamtlage« klargestellt, dass es in casu ausschließlich um Rechtsfindung auf Ebene der Vertragsverbindung (nicht: auf derjenigen der Einzelverträge) gehen kann, dass alles andere auf ein »Auseinanderreissen « einer sinnträchtigen Ordnung hinausgelaufen wäre. Wechselt man nun die Perspektive und betrachtet man BGE 115 II 452 nicht mehr mit der Brille des GouLDschen Kriteriums der »Agency«, sondern mit der Brille der »Efficacy«, tritt ein weiterer Aspekt der Vertragsverbindung in Erscheinung:

Das Urteil lässt deutlich werden, dass die Vertragsverbindung »innere Entwicklungsschranken« bzw. »constraints « kennt, die (ganz im Sinne GouLDs) die selektive Wirkung des Rechts in eine bestimmte Richtung kanalisieren: Die Vertragsverbindung sträubt sich gegen eine »Atomisierung « in einzelne Kontrakte und blockt gewissermassen das klassische Schuldrecht ab, das naturgemäß darauf aus ist, komplexe Sachverhalte in einzelne, bipolare und überschaubare Vertragseinheiten aufzuspalten. Im Ergebnis setzt sich die »Vernetzungs«-Logik durch, d.h. die Vertragsverbindung drängt sich dem Recht als eigenständige Institution (und nicht als zergliederungsfähige Kompilation) auf. Genau in diesen den Vertragsverbindungen eigenen »constraints « scheint das Moment auf, das Praxis und Lehre so verzweifelt mit den bekannten Annäherungsbegriffen $\mathrm{zu}$ erfassen suchen: »innere Bindung « oder »innerer Zusammenhang « von einheitlichen Verträgen ${ }^{44}$, »Finalnexus « auf der Ebene des Vertragsverbundes ${ }^{45}$, »identité d'objet « bzw. »communauté de cause ${ }^{46}$, »multilaterales Synallagma $\ll^{47}$, $»$ Innominatfigur ${ }^{48}$ usw. Was aber ist dieses Moment genau, das diese zwar suggestiven, aber letztlich doch unpräzisen Umschreibungen bezeichnen? Eine Gegenüberstellung von zwei neueren höchstrichterlichen Urteilen vom 21. Mai $2001^{49}$ und vom 16. Januar $2002^{50}$ ist in diesem Zusammenhang weiterführend:

Im erstgenannten Entscheid lagen Parteien im Streit, zwischen denen zwei separate Vereinbarungen, nämlich ein Miet- und ein Pachtvertrag, abgeschlossen worden waren. Diese Dauerschuldverhältnisse hatten ein eingerichtetes Restaurant zum Gegen-

44 Joachim Gernhuber (1973) »Austausch und Kredit im rechtsgeschäftlichen Verbund: Zur Lehre von den Vertragsverbindungen «, in: Gotthard Paulus, Uwe Diederichsen und ClausWilhelm Canaris (Hg.) Festschrift für Karl Larenz zum 70. Geburtstag, München: Beck, 455-494, 472; in diesem Sinne auch Ernst Kramer (1991) »Art. 19-22 OR«, in: Arthur Meier-Hayoz (Hg.) Berner Kommentar: Das Obligationenrecht, Bd. VI / 1. Abt. / 2. Teilbd. / Unterteilbd. 1a: Inhalt des Vertrages, Bern: Stämpfli, 33 N 64; Cerutti (Fn. 35) 6 f. N 19 ff.

45 Gernhuber (Fn. 44) 469 f.; ähnlich ders. (1989) Das Schuldverhältnis: Begründung und Änderung, Pflichten und Strukturen, Drittwirkungen, Tübingen: Mohr Siebeck, $710 \mathrm{f}$.

46 Bernard Teyssié (1975) Les groupes de contrats, Paris: LGDJ, 295; François Chaix (1995) Le contrat de sous-traitance en droit suisse: Limites du principe de la relativité des conventions, Basel: Helbing \& Lichtenhahn, $47 \mathrm{f}$.

47 Peter Heermann (1998) Drittfinanzierte Erwerbsgeschäfte: Entwicklung der Rechtsfigur des trilateralen Synallagmas auf der Grundlage deutscher und U.S.-amerikanischer Rechtsentwicklungen, Tübingen: Mohr Siebeck, 138 ff. et passim.

48 Schluep und Amstutz (Fn. 37) N 10.

49 BGE vom 21 Mai 2001, Nr. 4C.43/2000.

50 BGE vom 16. Januar 2002, Nr. 4C.288/2001. 
stand und waren insofern verbunden, als die Mieter / Pächter (ein Ehepaar) den Betrieb nur unter der Voraussetzung aufrechterhalten konnten, dass »l'ensemble des prestations prévues par les deux contrats, à savoir la mise à disposition des locaux, des équipements et de la licence d'alcool« vom Vermieter / Verpächter parallel und gleichzeitig erbracht wird ${ }^{51}$. Im Hinblick auf die Frage, ob die Mieter / Pächter den Pachtvertrag ordentlich beendigen können, ohne den Mietvertrag aufzulösen, hielt das Bundesgericht mit Bestimmtheit fest: »On se trouve ... en présence de deux contrats qui forment toutefois, dans l'idée des parties, une unité juridique et économique indissociable, au point de constituer un contrat unique mais complexe ... . Dans une telle situation aucun des contrats liés ne peut prendre fin séparément $\ll^{52}$. Die Begründung ist karg: Bezug genommen wird einerseits auf das (für die Lehre von der Vertragsverbindung re vera nicht weiterführende) Panic / Fleury-Urteil ${ }^{53}$, andererseits auf eine Lehrmeinung von ENGEL, der sich aber an der angegebenen Stelle zur Frage der Beendingung verbundener (Dauer-) Verträge nicht unmittelbar äußert ${ }^{54}$.

Im zweitgenannten Entscheid war ein Werkvertrag zwischen H. (Besteller) und der W. AG (Unternehmerin) zu beurteilen, der den Bau von 56 Einfamilienhäusern betraf. Im Laufe der Zeit war dieser Vertrag dem H. unliebsam geworden und er versuchte sich seiner zu entledigen. Zu diesem Zweck trug er vor, dass der Werkvertrag an einen (nie zustande gekommenen) Kaufvertrag gekoppelt war, mit dem sich die W. AG verpflichtet hätte, eines der zu erstellenden Objekte zu erwerben. Der Umstand, dass der beabsichtigte Kaufvertrag nie geschlossen worden sei, habe - so H. weiter - die Nichtigkeit des Werkvertrages zur Folge, da sich beide Vereinbarungen als zusammengesetzte Verträge im Sinne einer Gültigkeitsvoraussetzung gegenseitig bedingen würden. Obwohl unbestritten geblieben ist, dass die Parteien den erwähnten Kaufvertrag ernsthaft ins Auge gefasst hatten, lehnte das Bundesgericht die Argumentation des H. ab. Auch hier ist es der weiteren Analyse dienlich, die Urteilsbegründung im Original wiederzugeben:

»Nach den Feststellungen der Vorinstanz einigten sich die Parteien zunächst über den Abschluss des Werkvertrags, wobei sie übereinstimmend der Auffassung waren, dass die Klägerin später eines der noch zu erstellenden Häuser samt Autoeinstellplatz zu einem bereits festgelegten Preis kaufen werde. Warum unter diesen Umständen ein zusammengesetzter Vertrag gegeben sein soll, wird im angefochtenen Urteil nicht gesagt. Es ist denn auch fraglich, worin das Austauschverhältnis zwischen den gegenseitigen Verpflichtungen aus den beiden Verträgen liegen soll. Wie aus dem Urteil [der Erstinstanz] ... hervorgeht, ist offenbar die Höhe des Werklohnes im Hinblick auf die Höhe des Kaufpreises im später abzuschließenden Kaufvertrag festgesetzt worden. Das reicht indessen für eine Vertragsverbindung ... nicht aus. Vielmehr liegt der Fall vor, dass die Preiskalkulation in der Erwartung erfolgt, aus der zukünftigen geschäftli-

51 BGE vom 21 Mai 2001, Nr. 4C.43/2000, E. 2.d).

52 BGE vom 21 Mai 2001, Nr. 4C.43/2000, E. 2.d).

53 BGE 107 II 144 E. 2.

54 Pierre Engel (2000) Contrats de droit suisse: Traité des contrats de la partie spéciale du Code des obligations, de la vente au contrat de société simple, articles 184 à $551 \mathrm{CO}$, ainsi que quelques contrats innommés, 2. Aufl., Bern: Stämpfli, $741 \mathrm{f}$. 
chen Verbindung werde sich ein gewisser Ausgleich ergeben. Ein Austauschverhältnis zwischen den gegenseitigen Verpflichtungen aus Werk- und Kaufvertrag ist damit aber nicht gegeben ${ }^{55}$.

Im Grunde genommen liefert das Bundesgericht im zweiten Urteil die Begründung für das erste nach: Während die Erwägungen im Restaurant-Fall auch dahingehend gelesen werden könnten, dass die Frage, ob eine Vertragsverbindung vorliegt, vom Dispositionswillen der Parteien (»... dans l'idée des parties ...«) abhängt, stellen die Erwägungen im Baukomplex-Fall klar, dass der Parteikonsens für die Verkoppelungsfrage nicht ausschlaggebend sein $\mathrm{kann}^{56}$. Danach vermag ein unspezifizierter Konsens der Parteien, die abgeschlossenen Vereinbarungen würden irgendwie zusammenhängen (z.B. dadurch - wie das im zweiten Urteil der Fall war -, dass beabsichtigt ist, die Preiskalkulationen im Werk- und im Kaufvertrag auf einer Gesamtbasis vorzunehmen), nicht auszureichen, um das Schuldrecht von seiner partikularistischen Perspektive abzubringen. Vielmehr muss nach höchstrichterlicher Ansicht zwischen den mehreren Vereinbarungen ein Konnex sozusagen »in der Sache« bestehen, d.h. ein Konnex, der sich aus den Umständen des Vertragsschlusses (bzw. der Vertragsschlüsse) ergibt. Aber wie muss dieser Konnex beschaffen sein? Die Antwort des Bundesgerichts scheint mir deutlich: Nur insoweit das auf mehrere bilaterale Versprechen gestützte »Gesamtprojekt « der Art ist, dass seine Realisierung die Erfüllung sämtlicher Versprechen als conditio sine qua non voraussetzt, sei eine Abweichung von einem »linearen« zugunsten eines »vernetzten«Vertragsdenkens gerechtfertigt. Damit wird nachvollziehbar, weshalb die Höchstinstanz in den hier fraglichen Urteilen jeweils zu diametral entgegengesetzten Ergebnissen gekommen ist, obwohl in beiden Fällen Willenselemente der Parteien vorlagen, die auf eine Koppelung der Vereinbarungen hindeuteten: Im ersten Fall hing der Betrieb des Restaurants unabdingbar von der Erfüllung sowohl des Miet- als auch des Pachtvertrages ab; anders im zweiten, in dem die 56 Einfamilienhäuser durchaus auch ohne den anvisierten Kaufvertrag zwischen H. und der W. AG hätten erstellt werden können. Aus diesen Beobachtungen ergeben sich wichtige Rückschlüsse für die Ontogenetik der Vertragsverbindung:

Erst wenn sich der Wille der Parteien und die Umstände in der Weise kombinieren, dass es zwischen mehreren Verträgen zur Ausbildung eines Konnexes der beschriebe-

55 BGE (Fn. 50) E. 2.

56 Diese Klarstellung ist an Bedeutung nicht zu überschätzen: Entgegen einer Tendenz in der Lehre (vgl. Walter Schluep (2003) »Zusammengesetzte Verträge: Vertragsverbindung oder Vertragsverwirrung?«, in: Heinrich Honsell, Wolfgang Portmann, Roger Zäch und Dieter Zobl (Hg.) Aktuelle Aspekte des Schuld- und Sachenrechts: Festschrift für Heinz Rey zum 60. Geburtstag, Zürich: Schulthess, 288-306, 304 ff.), die Vertragsverbindung von einer sog. Koppelungsabrede abhängig zu machen, zeigen die Ausführungen des Bundesgerichts nicht nur, dass es einer solchen Abrede nicht notwendig bedarf, um die Anwendbarkeit der Lehre vom zusammengesetzten Vertrag zu begründen. Den zwei im Text gegenübergestellten Urteilen muss darüber hinaus auch noch entnommen werden, dass der Parteiwille bei der Begründung einer Vertragsverbindung nur eine untergeordnete Rolle spielt. »Untergeordnet « in dem Sinne, dass es zwar unmöglich zu Verträgen kommen kann, ohne dass diese vom Parteikonsens getragen werden; dass aber die Verbindung dieser Verträge stets von Umständen abhängt, die die Parteien nicht zwangsläufig reflektiert haben müssen. 
nen Qualität kommt, bestehen im polyvertraglichen Gebilde »innere Entwicklungsschranken« oder »constraints«, die den Selektionsfaktoren in der Umwelt dieses Gebildes entgegenwirken und die Effekte dieser Faktoren verändern oder sogar beseitigen. Diese »constraints « sind das Produkt des von jedem Vertrag, der Bestandteil des rechtsgeschäftlichen Netzwerkes bildet, verlangten Beitrags zur Stabilisierung des Ganzen (das im Grunde genommen als solches äußerst instabil ist, weil es von den jeweils kontradiktorischen Sichten der Einzelverträge geprägt ist ${ }^{57}$ ). Die einzelnen Verträge zielen deshalb nicht, wie das sonst in kontraktuellen Situationen zutrifft, auf die Herstellung einer in sich kohärenten, aber in dieser Kohärenz abgeschlossenen Ordnung, sondern auf Beiträge zur Bildung einer konsistenten höheren Ordnung, die ein die jeweiligen »Projekte« der Verbundveträge übersteigendes »Gesamtprojekt« zum Gegenstand hat. Diese »Stabilisierungsbeiträge« sind evolutorische Kräfte, die im Sinne der GouLDschen »constraints « neben der darwinistischen natürlichen Selektion wirken und den Vertragsverbund im Wettbewerb der verschiedenen Formen institutioneller Arrangements (Vertrag, Personengesellschaft, Körperschaft, Anstalt usw.) als eigenständige institutionelle Form durchsetzen.

Fraglich bleibt, ob damit die Vertragsverbindung evolutionstheoretisch umfassend beleuchtet ist. Das dritte GoulDsche Kriterium des »Scope« weist darauf hin, dass im Prozess der Evolution zuweilen nicht-evolutorische Ereignisse (wie Katastrophen welcher Art immer) einen maßgeblichen Einfluss auf die Formen des Lebens ausüben können. Und gerade solche Ereignisse müssen für eine Abrundung des ontogenetischen Bildes der Vertragsverbindung in Betracht gezogen werden. Das Stichwort lautet: Netzwerkrevolution ${ }^{58}$. Überall in der Gesellschaft werden seit einiger Zeit heterarchische Phänomene registriert und das Vertragsrecht bleibt davon nicht verschont. Im Gegenteil: Die »Heterarchisierung « der sozialen Welt scheint in gewissen Lebensbereichen die Vertragsverbindung als neue Organisationsform drastisch zu fördern, ja zuweilen sogar als unabwendbar aufzudrängen und auf Dauer zu stellen.

Zeugnis dafür legt der vielbeachtete und dogmatisch höchst umstrittene BGE 121 III $310 \mathrm{ab}^{59}$. In diesem Urteil hatte sich das Bundesgericht mit einer Geldüberweisung zu

57 Vgl. zu diesem Punkt Vincent Lemieux (1999) Les réseaux d'acteurs sociaux, Paris: PUF, $17 \mathrm{f}$.

58 Vgl. z.B. Albert-László Barabaási (2003) Linked: How Everything Is Connected to Everything Else and What It Means for Business, Science, and Everyday Life, New York: Plume.

59 Vgl. vor allem Walter Fellmann und Jörg Schwarz (1996) »Geldüberweisung mit Hilfe des Bankenclearingsystems; vertraglicher Direktanspruch des Überweisenden gegen die sich weisungswidrig verhaltende Empfängerbank (Art. 32, 112, 127, 398 Abs. 3 OR): Bundesgericht, I. Zivilabteilung, 27.6.1995, T. c. Bank X, Berufung (= BGE / ATF 121 III 310)«, 5 AJP, 97 ff.; Martin Hess (1996) »Bargeldlose Überweisung mit Hilfe von Interbankzahlungssystemen - Vertrauenshaftung im Zahlungsverkehr? BGE 121 III 310«, 14 recht, 144 ff.; Wolfgang Wiegand (1997) »Die privatrechtliche Rechtsprechung des Bundesgerichts im Jahre 1995«, 133 ZBJV, 110-135, 123 ff.; Hanspeter Dietzi (2000) »Zahlungsverkehr«, in: Wolfgang Wiegand (Hg.) Berner Bankrechtstag 2000: Rechtliche Probleme des Zahlungsverkehrs, Bern: Stämpfli, 139-177, 161 f.; Sébastien Bettschart (2000) Virement en chaîne et assignation bancaire: Droit suisse des obligations et contexte international, Zürich: Schulthess, 284 ff. 
befassen, die mit Hilfe des schweizerischen Bankenclearingsystems (Swiss Interbank Clearing; SIC) ausgeführt wurde ${ }^{60}$. T. hatte B. ein Darlehen von CHF 300`000.- in Aussicht gestellt und sich bereit erklärt, den Betrag sogleich (also noch vor Abschluss des Darlehensvertrags) auf ein Sperrkonto bei der Bank Y. (Empfängerbank) zu überweisen. Mit dieser Überweisung beauftragte T. die Bank Z. (Absenderbank), die vertragsgemäß handelte und der Empfängerbank gegenüber die Begünstigten mit der Formel »B. \& T. Sperrkonto« umschrieb. In der Folge blieb der Abschluss des Darlehensvertrages zwischen T. und B. aus. Trotzdem gab B. ohne jegliche Mitwirkung des T. den »gesperrten« Betrag frei, was die Empfängerbank auch ausführte, obwohl bankintern das Konto die Bezeichnung »Sperrkonto B. \& T.«trug ${ }^{61}$. T. machte gegen die Empfängerbank einen vertraglichen Anspruch auf Schadenersatz geltend und wurde darin vom Bundesgericht geschützt.

Die Reaktion der Lehre auf diese Entscheidung war einmütig. Stellvertretend für sie sei FELLMANN und SCHWARZ das Wort erteilt: »Das Urteil ... ist im Ergebnis sicher richtig. Seine Begründung vermag aber nicht zu überzeugen. ... Man erhält den Eindruck, ausschlaggebend für das Dispositiv seien nicht rationale Überlegungen, sondern ein mehr oder weniger bestimmtes , Rechtsgefühl ${ }^{\text {‘ gewesen }}{ }^{62}$. »Irrationalität $«$ der Argumentation, »Rechtsgefühl « als Entscheidungsmotiv, oder gar: »richtiges Ergebnis « / »falsche Begründung ${ }^{63}$ - das weckt Neugier, und nicht bloß aus juristischer, sondern auch aus rechtssoziologischer Sicht. Halten wir uns aber vorerst einmal bei dieser anscheinend so verfehlten Urteilsbegründung auf. Ausgehend von der Beobachtung, dass das SIC »als Girosystem im Dienste des mehrgliedrigen Überweisungsverkehrs [steht] ... [und] eine zentral gesteuerte und damit schnelle Abwicklung von Kettenüberweisungen [ermöglicht], die ihren Grund darin haben, dass der Überweisungsempfänger sein Konto nicht bei der gleichen Kontostelle unterhält wie der Überweisende ${ }^{64}{ }^{6}$, deckt das Bundesgericht in zwei Schritten die schuldrechtliche Organisation einer Geldüberweisung mit Hilfe eines Interbankzahlungssystems auf:

Zunächst erfolgt eine allgemeine Qualifikation der zwischen den Beteiligten bestehenden Rechtsbeziehungen: »Im mehrgliedrigen Überweisungsverkehr handeln die zwischengeschalteten Banken in eigenem Namen, aber auf fremde Rechnung, somit als indirekte Stellvertreterinnen. Eine dergestalt vorgenommene Überweisung wird mittels mehrerer, kettenartig verbundener Verträge abgewickelt, an denen unterschiedliche Parteien beteiligt sind, wobei die Relativität der jeweiligen Rechtsbeziehungen zu beachten ist. So besteht zwischen dem Überweisenden und der Erstbank ein Girovertrag, auf den die Regeln des Auftragsrechts Anwendung finden. Der in diesem Rahmen erfolgende Vergütungsauftrag ist eine an die Erstbank gerichtete Weisung (Art. 397 OR) des Inhalts, mit der kontoführenden Bank des Empfängers ein Anweisungsverhältnis im Sinne von Art. 466 ff. OR einzugehen. Die beteiligten Banken sind sodann unter

60 Vgl. zum Swiss Interbank Clearing statt anderer Bettschart (Fn. 59) 35 ff. m.w.N.

61 Vgl. die ausführlichere Sachverhaltsdarstellung in BGE $121 \mathrm{III} 310 \mathrm{f}$.

62 Fellmann und Schwarz (Fn. 59) 97.

63 So Hess (Fn. 59) 155.

64 BGE 121 III 312 E. 3. 
sich durch selbständige Giroverträge verbunden, auf die ebenfalls die Regeln des Auftragsrechts anwendbar sind. Aus alldem ergibt sich, dass zwischen dem Überweisenden und der Empfängerbank grundsätzlich keine unmittelbaren Vertragsbeziehungen bestehen ${ }^{65}$. Sodann unternimmt das Bundesgericht eine Qualifikation des Dreiecksverhältnisses Überweisender (T.) / Absenderbank (Bank Z.) / Empfängerbank (Bank Y.) und erblickt darin eine befugte Substitution im Sinne von Art. 398 Abs. 3 OR: »Die Erstbank [sc. die Bank Z.] erhielt vom Kläger [sc. T.] den Auftrag, den Begünstigten ,B. \& T. ' Fr. 300‘000.- auf ein ,Sperrkonto“ bei der Bank Y. zu überweisen. Der Kläger durfte nach dem Vertrauensprinzip erwarten, dass auch der Vollzug der Gutschrift auf dem ,Sperrkonto“ zum Vertragsinhalt gehörte. Die Erstbank war deshalb gegenüber dem Kläger nicht nur dazu verpflichtet, der Empfängerbank seine Zahlungsbereitschaft anzuzeigen, sondern auch, die Gutschrift auf dem angegebenen Konto zu veranlassen. Die Gutschrift konnte sie aus rechtlichen Gründen nicht selbst vornehmen, sondern sie musste die kontoführende Empfängerbank damit beauftragen; zur Erreichung des Vertragsziels und Erfüllung eines Teils des Vertrages somit im Interesse des Auftraggebers eine am Vertrag nicht beteiligte Drittpartei beiziehen. Unter diesen Umständen ist die Empfängerbank als Substitutin im auftragsrechtlichen Sinn zu betrachten $\aleph^{66}$.

Nach dieser Flurbereinigung schreitet das Bundesgericht zur Prüfung der eigentlich interessierenden Frage: Steht T. eine Schadenersatzforderung aus Vertrag gegen die Bank Y. zu ${ }^{67}$ ? Der Aufbau der Argumentation entspricht, wie vor allem WIEGAND no$\operatorname{tiert}^{68}$, nicht dem sonst Üblichen, was, wie später noch näher zu erläutern sein wird, vorliegend von besonderer Bedeutung ist. Weil ein mandatsrechtliches Substitutionsverhältnis gegeben ist, knüpft das Bundesgericht an die Frage an, ob dem T. eine Schadenersatzforderung aus Art. 399 Abs. 3 OR erwachse, der dem Hauptauftraggeber einen Direktanspruch gegen den Substituten einräumt. Indessen umfasst dieser Anspruch nach dem Wortlaut der Bestimmung inhaltlich nur die Ansprüche, die dem Beauftragten gegen den Substituten zustehen. In casu hilft das ersichtlich nicht weiter, weil der Schaden, den T. gegen die Bank Y. geltend machen will, nur bei ihm und nicht beim Beauftragten (der Bank Z.) eingetreten ist ${ }^{69}$. Dead end? Keineswegs: Das

65 BGE 121 III 312 f. E. 3.

66 BGE 121 III 324 E. 4; in der Lehre ist diese Auffassung nicht unbestritten; vgl. dazu die Übersicht bei Dietzi (Fn. 59) 161 m.w.N.

67 Diese Frage stellte sich vor allem deshalb, weil eine deliktische Forderung - die von der Vorinstanz im Grundsatz bejaht wurde - verjährt war; vgl. BGE 121 III 312.

68 Wiegand (Fn. 59) 126.

69 Vgl. etwa Franz Werro (2003)»Art. 399«, in: Luc Thévenoz und Franz Werro (Hg.) Commentaire Romand: Code des obligations I (Code des obligations art. 1-529; Loi sur le crédit à la consommation, Loi sur les voyages à forfait), Genève: Helbing \& Lichtenhahn, $2061 \mathrm{~N}$ 6; meistens wird dieser Punkt in der Literatur heute nicht mehr besonders begründet und im Anschluss an BGE 121 III 310 festgehalten, der Anspruch des Hauptauftraggeber nach Art. 399 Abs. 3 OR umfasse auch den eigenen Schaden; vgl. etwa repräsentativ Rolf Weber (2003) »Art. 399 OR «, in: Heinrich Honsell, Nedim Vogt und Wolfgang Wiegand (Hg.) Basler Kommentar zum Schweizerischen Privatrecht: Obligationenrecht I (Art. 1-529 OR), 3. Aufl., Basel: Helbing \& Lichtenhahn, 2189 N 6 m.w.N. In der Sache scheint dieser Anspruch also kaum mehr strittig zu sein; nur noch dogmatisch wird nach seiner Fundierung gesucht. 
Bundesgericht stellt fest, dass es die Lehre »durchwegs « befürworte, den direkten Anspruch des Auftraggebers gegen den Substituten unabhängig davon zu gewähren, ob dieser den Beauftragten durch sein Verhalten geschädigt habe ${ }^{70}$. Allerdings leite nur eine Minderheit diesen Direktanspruch aus Art. 399 Abs. 3 OR mit dem Argument ab, diese Vorschrift sei als gesetzlich geregelter Fall der Drittschadensliquidation zu verstehen $^{71}$. Die Mehrheit erblicke die rechtliche Grundlage des Anspruchs im Vertragsverhältnis zwischen dem Beauftragten und dem Substituten, dies mit der Begründung, dieses Verhältnis sei entweder als Vertrag zugunsten eines Dritten (Art. 112 OR) ${ }^{72}$ oder als Vertrag mit Schutzwirkung für Dritte zu qualifizieren ${ }^{73}$. Nach rechtsvergleichenden Erwägungen, in denen dargetan wird, dass in den Nachbarländern ein Direktanspruch des Hauptauftraggebers gegenüber dem Substituten insbesondere für den Fall der mehrgliedrigen Banküberweisung anerkannt wird ${ }^{74}$, hätte man eine höchstrichterliche Stellungnahme für eine der drei möglichen dogmatischen Konstruktionen erwartet. Diese blieb aus - und darin liegt wohl das Außergewöhnliche an BGE 121 III 310, das es zu verstehen gilt. Im Ergebnis bejaht das Bundesgericht den fraglichen Direktanspruch ohne Dogmatik, aber mit der Verweisung auf faktische Bedürfnisse des Rechtsverkehrs (daher auch der weitverbreitete Eindruck, diese Entscheidung sei aus dem »Rechtsgefühl« erwachsen - was auch immer damit gemeint ist). Solche Bezugnahmen auf Faktizitäten sind in der höchsten Zivilrechtsprechung alles andere als alltäglich, erst recht, wenn sie dann noch, wie im hier fraglichen Urteil, als rationes decidendi fungieren. Die entscheidende Passage lautet:

»Zu berücksichtigen ist ..., dass sich die Anerkennung eines Direktanspruchs ... aufgrund von ... Überlegungen aufdrängt, die unabhängig von der Frage der Anwendbarkeit von Art. 399 Abs. 3 OR unmittelbar auf die rechtliche Wertung der Interessen der am Überweisungsverhältnis beteiligten Parteien abstellen. Als Ausgangspunkt dient der Umstand, dass die indirekten Vertretungsverhältnisse im mehrgliedrigen Überweisungsverkehr regelmäßig offenliegen, weil keine der beteiligten Banken davon ausgehen darf, die andere handle ausschließlich auf eigene Rechnung. Das Drittinteresse ist dem bankinternen Giroverkehr immanent und allseits erkennbar, ebenso das Schutzbedürfnis des Überweisenden gegenüber Fehlleistungen der Banken. Der bankinterne Giroverkehr steht im Dienste der Überweisungspartner und soll die Geschäftsabwicklung zwischen den Banken erleichtern. Die mit dieser Erleichterung einhergehenden Risiken von Fehlleistungen aber müssen sachgerecht die Banken und nicht die Über-

70 BGE 121 III 315 E. 4.a).

71 BGE 121 III 315 E. 4.a).

72 BGE 121 III 315 E. 4.a); vgl. in diesem Sinne (nebst dem a.a.O. zitierten Schrifttum) Pierre Tercier (2003) Les contrats spéciaux, 3. Aufl., Zürich: Schulthess, 672 N 4661; kritisch gegenüber dieser Konstruktion (mit einleuchtenden Argumenten) Fellmann und Schwarz (Fn. 59) 98.

73 BGE 121 III 315 E. 4.a); diese Konstruktion, die vor Bundesgericht vom Referenten vorgeschlagen, aber in den Urteilserwägungen stillschweigend abgelehnt wurde, ist in der Literatur auf positives Echo gestossen; vgl. nur Fellmann und Schwarz (Fn. 59) 99; Wiegand (Fn. 59) 127.

74 BGE 121 III 315 ff. E. 4.b). 
weisungspartner tragen. Diese dürfen nicht allein wegen der Zwischenschaltung einer weiteren Bank schutzlos bleiben, obwohl die Voraussetzungen einer Pflichtverletzung an sich vorliegen. Es geht letztlich darum, zu verhindern, dass aufgrund rein zahlungstechnischer oder organisatorischer Zufälligkeiten Schutzansprüche wegfallen bzw. Pflichten leerlaufen, die ,eigentlich', das heißt abgesehen von der Vertragsgläubigerstellung des Geschädigten, gegeben sind .... «"

Leicht zu erkennen ist, dass mit diesen Ausführungen der richterlich gewährte Anspruch dogmatisch im Vagen bleibt. Ob sich in dieser Hinsicht ein tragfähiges Fundament finden lässt, ist eine Frage der Innenperspektive des Rechts, die erst im nächsten Abschnitt zur Diskussion steht. Worauf es im Kontext ankommt, ist der Umstand, dass das Bundesgericht offenbar spürte, dass die Lösung nicht auf klassisch-einzelvertraglicher Ebene, sondern unmittelbar auf der emergenten Ebene des Vertragsverbundes zu suchen ist. Und zwar in einem ganz spezifischen Sinne, der evolutionstheoretisch nahelegt: Die Alternative, den Sachverhalt auf der Grundlage traditioneller schuldrechtlicher Überlegungen zu behandeln, bestand im Grunde gar nicht; sie wurde von der Institutionalisierung des SIC (als »katastrophistisches« Ereignis) »zerstört«. Die Weigerung des Bundesgerichts, das befürwortete Ergebnis auf den Rechtsfiguren der Drittschadensliquidation, des Vertrages zugunsten eines Dritten oder des Vertrages mit Schutzwirkungen für Dritte abzustuitzen, kann also (auch) dahin gedeutet werden, dass es der Ansicht war, die damit verfolgten, durch und durch »bipolaren « Policy-Strategien würden auf die emergente Ordnung der mehrgliedrigen Banküberweisung nicht $»$ passen $\aleph^{76}$. BGE 121 III 310 lehrt somit, dass das Auftreten von Vertragsnetzen in der Wirtschaft nicht nur das Ergebnis selektiv wirkender Umweltkräfte und »innerer Entwicklungsschranken« sein kann, sondern auch die Folge radikaler Umbrüche in der Lebenswelt. »Katastrophismus « spielt also auch in der Evolution der institutionellen Arrangements mit.

\section{III.}

Die anhand des GoulDschen Analyserasters (Agency / Efficacy / Scope) durchgeführte evolutionstheoretische Beobachtung institutioneller Arrangements, die auf einer Mehrheit von vernetzten Verträgen aufgebaut sind, hat dreierlei gezeigt: (1) Zunächst einmal, dass sich aus solchen Vertragsvernetzungen emergente Ordnungen bilden können, die sich von den »bipolaren« Ordnungen der Einzelverträge unterscheiden und nach einer eigenen Logik (einer »Netzwerk «-Logik) operieren. (2) Diese Arrangements sind sodann (unter anderem) den selektiven Kräften des Vertragsrechts ausgesetzt, die dazu tendieren, vernetzte Verträge nicht in ihrer Verbundenheit anzugehen, sondern sie als »autonome« Einheiten zu behandeln. Erlangen vernetzte Verträge in

75 BGE 121 III 317 E. 4.c).

76 Eine eingehende Auseinandersetzung mit dieser Frage - namentlich mit den Defiziten der klassischen schuldrechtlichen Modelle zur Bewältigung vertragsübergreifender Leistungsstörungen - findet man bei Matthias Rohe (1998) Netzverträge: Rechtsprobleme komplexer Vertragsverbindungen, Tübingen: Mohr Siebeck, 65 ff. 
ihren gegenseitigen Bezügen allerdings eine solche Organisationsdichte, dass sich der eigentliche Sinn der Parteivereinbarungen nicht mehr auf der Ebene der jeweils ausgetauschten Willenserklärungen, sondern auf derjenigen ihrer Koppelung - man könnte auch sagen: auf der Ebene ihrer »Symbiose« zur Vertragsverbindung - befindet, so bilden sie »constraints« heraus, die dem erwähnten Reflex des Vertragsrechts sehr effektiv entgegenwirken. Eine solche Bildung von »constraints« findet namentlich dann statt, wenn die Verträge des rechtsgeschäftlichen Verbundes derart verquickt sind, dass die Realisierung eines »Projektes«, das ihnen $»$ gemein $«$ ist $^{77}$, die Erfüllung sämtlicher versprochener Leistungen unabdingbar voraussetzt. (3) In gewissen Lebensbereichen haben heute sozioökonomische oder technologische Umbrïche eine solche Bedeutung erlangt, dass sich die Wahl zwischen einer einzelvertraglichen oder einer verbundmäßigen Sicht schon gar nicht mehr anbietet. In solchen »katastrophistischen « Fällen haben die Einzelverträge, die Bestandteile des rechtsgeschäftlichen Verbundes sind, ihre Autonomie (ihren »Eigensinn «) »verloren«. Die Verselbständigung der Ordnung, die aus dem Aggregat von bipolaren Vereinbarungen fließt, zeigt sich hier mit besonderer Deutlichkeit und es fragt sich, wie das Recht darauf reagieren soll.

Dieser Frage ist der vorliegende Abschnitt gewidmet. Um sie anzugehen, sind zwei Schritte zu vollziehen: (1) Als erstes muss in Erfahrung gebracht werden, worin überhaupt die Ursache dafür liegt, dass das klassische Vertragsrecht erhebliche Mühe bekundet, den Sachverhalt der Vertragsverbindung mit den dogmatisch gängigen Rechtsfiguren einzufangen. Wir wissen zwar, dass das Problem in seiner (historisch bedingten) Neigung besteht, institutionelle Arrangements interpretativ in einzelne Rechtsgeschäfte, die in sich geschlossen sind, zu reduzieren. Aber das bleibt eine AuBenperspektive des Schuldrechts, die im vorausgegangenen Abschnitt eingenommen wurde. Worum es vorliegend geht, ist die Beleuchtung des Schuldrechts von »Innen« her. Demnach lautet die Frage jetzt: Weshalb zeigt sich das Vertragsrecht in seinen internen Operationen so verwirrt, wenn es mit Vertragsverbindungen konfrontiert wird? Aus welchem Grund muss es, wie vor allem die Analyse von BGE 121 III 310 gezeigt hat, auf Lösungsansätze zurückgreifen, die es am Maßstab der anerkannten Dogmatik gemessen nicht zu begründen vermag? (2) Sodann ist zu fragen, wie die Erkenntnisse, die aus der durchgeführten evolutionstheoretischen Beobachtung von Vertragsverbindungen gewonnen wurden, in das Vertragsrecht »eingebracht« werden können, und vor allem: $\mathrm{Zu}$ welchen dogmatischen Lösungen, die mit dem positiven Recht kompatibel sind, regen diese Erkenntnisse an?

Im Hinblick auf den ersterwähnten Punkt gehe ich von der These aus, dass Vertragsverbindungen unmittelbar mit dem Phänomen der funktionalen Differenzierung der Gesellschaft zusammenhängen und dass gerade dieser Zusammenhang das positive

77 Mit dem Begriff »gemein « wird keineswegs impliziert, dass die Parteien einen gemeinsamen Zweck (im Sinne des Gesellschaftsrechts) verfolgen. Vertragsverbindungen ist gerade dieses Element fremd, da sie sich aus kontradiktorisch angelegten Einheiten, nämlich bipolaren Verträgen, zusammensetzen. Kurzum: Das »gemeine " Projekt, das im Text angesprochen ist, ist ein solches, das strukturell von den Verträgen, nicht von allen Parteien getragen wird. 
Vertragsrecht überfordert ${ }^{78}$. Um diese These zu begründen, muss die differenzierungstheoretische Dimension der Institution des Vertrages offengelegt werden. Aus systemtheoretischer Perspektive ist der Vertrag nicht konsensualer Akt zwischen mehreren Akteuren, sondern strukturelle Kopplung zwischen Funktionssystemen der Gesellschaft (Wirtschaft, Recht, Wissenschaft usw.) ${ }^{79}$. Ausgesagt wird damit im wesentlichen, dass der Vertrag die beteiligten Systeme zur wechselseitigen Beschreibung und Beobachtung befähigt ${ }^{80}$. Das wird dadurch vollbracht, dass im Vertrag (als Form der strukturellen Kopplung) Möglichkeiten gespeichert sind, die die beteiligten Systeme als Information verwenden können. LUHMANN unterstreicht, dass unter diesem Gesichtspunkt »strukturelle Kopplung einerseits einen Ausschließungseffekt hat - in diesem Bereich ist das System indifferent - und andererseits eine Kanalisierung von Kausalitäten bewirkt, die das System verwenden kann ${ }^{81}$. Damit stellt sich die Frage, wie die aus dem Vertrag gewonnenen Informationen in den beteiligten Systemen genau benutzt werden. In diesem Zusammenhang ist zutreffend darauf hingewiesen worden, dass diese Informationen »insofern konstitutiv für ein Sozialsystem [sind], als ... [sie] latente Erwartungen in tatsächliche Verpflichtungen, bloße Projektionen in Operationsbindungen umwandel[n] $\ll^{82}$. Anders gewendet erzeugen diese Informationen im betreffenden Funktionssystem die Strukturen eines Subsystems, die »auf Erfüllung des Vertragszweckes, drängen $\ll^{83}$. Hier wird nun auch die Fragmentierung des Vertrages im Kontext der sozialen Differenzierung voll sichtbar: Über diese Institution entstehen nämlich in den beteiligten Systemen jeweils autonome Diskurse, die je nach Eigenlogik des in Frage stehenden Bereichs unterschiedliche Gestalten annehmen. Der Vertrag führt z.B. (1) in der Wirtschaft zur ökonomischen Transaktion als organisiertes Verfügungsverfahren ${ }^{84}$, (2) im Recht zu einem normativen Diskurs im Rahmen des Vertrages »als Gefüge und als Prozess ${ }^{85}$, (3) in anderen Funktionssystemen (Wissenschaft, Kunst, Medizin, Erziehung usw.) zu einem Projekt, das in seiner kommunikati-

78 Vgl. eingehend Marc Amstutz (2003)»Vertragskollisionen: Fragmente für eine Lehre der Vertragsverbindung «, in: Heinrich Honsell, Wolfgang Portmann, Roger Zäch und Dieter Zobl (Hg.) Aktuelle Aspekte des Schuld- und Sachenrechts: Festschrift für Heinz Rey zum 60. Geburtstag, Zürich: Schulthess, 161-176, 165 ff. m.w.N.; vgl. auch generalisierend Niklas Luhmann (2000) Organisation und Entscheidung, Opladen: Westdeutscher Verlag, 411.

79 Vgl. etwa Luhmann (Fn. 78) 440 ff.; Peer Zumbansen (2000) Ordnungsmuster im modernen Wohlfahrtsstaat: Lernerfahrungen zwischen Staat, Gesellschaft und Vertrag, BadenBaden: Nomos, 206; Tania Lieckweg (2003) Das Recht der Weltgesellschaft: Systemtheoretische Perspektiven auf die Globalisierung des Rechts am Beispiel der lex mercatoria, Stuttgart: Lucius \& Lucius, $45 \mathrm{ff}$.

80 Zumbansen (Fn. 79) 206; Lieckweg (Fn. 79) 46 ff.

81 Niklas Luhmann (2002) Einführung in die Systemtheorie, Heidelberg: Carl-Auer-Systeme, 121.

82 Gunther Teubner (1998) »Vertragswelten: Das Recht in der Fragmentierung von Private Governance Regimes «, 17 RJ, 234-265, 249.

83 Teubner (Fn. 82) 249.

84 Lieckweg (Fn. 79) 46.

85 Karl Larenz (1987) Lehrbuch des Schuldrechts: Erster Band: Allgemeiner Teil, 14. Aufl., München: Beck, 26 ff. 
ven Durchführung die betreffende Produktionslage im System (Erkenntnisgewinn, ästhetischer Mehrwert, Gesundheitsverbesserung, Sozialisierungs- und Kompetenzsteigerung usw.) verändert ${ }^{86}$. Und dies alles simultan!

Stellt man diese $»$ gesellschaftliche Vieldimensionalität des einen Vertrages ${ }^{87}$ in Rechnung, wird es möglich, die konkreten Probleme, die Vertragsverbindungen im System des Vertragsrechts auslösen, zu rekonstruieren. Denn dann wird einmal erkennbar, dass der Vertrag in den Funktionssystemen der Gesellschaft zur Emergenz von Diskursen beiträgt, die nicht auf der Stufe der einfachen Interaktion stecken bleiben, sondern zuweilen eine Komplexität erlangen, die sie zur Entwicklung von Reflexionsfähigkeit nötigt, also zu reflexiven (Teil-) Systemen verdichtet. Denkbar ist das vor allem in Fällen langfristiger Kontrakte (Franchise, Just-in-time, Joint Venture, Grossbauten, Technologietransfer usw.), bei denen die Beziehungen zwischen den Parteien nicht mehr von der Begrenztheit und der Kurzfristigkeit der spot transaction geprägt sind; diese Beziehungen weisen dann vielmehr die Qualität enger Kooperationsverhältnisse auf. Damit beginnen die aus der strukturellen Kopplung »Vertrag « hervorgehenden (Teil-) Systeme ihre Umwelt zu beobachten bzw. zu reflektieren, und das heißt insbesondere: die möglichen Konflikte zwischen ihnen und den Systemenin-ihrer-Umwelt zu antizipieren. Diese Konflikte werden mittels Reflexion im betreffenden (Teil-) System mit Blick auf ihre Folgen eingeschätzt und für interne Korrekturen der Handlungssteuerung ausgewertet. Lösen nun mehrere Verträge in den durch sie gekoppelten Funktionssystemen jeweils Diskurse aus, die reflexive Komplexität ausbilden, so kann es dazu kommen, dass diese Diskurse sich gegenseitig beobachten und (im beschriebenen Sinne) reflexiv Rechnung tragen. M.a.W. kommt es dann zur Verschleifung von unterschiedlichen Diskursen und genau diese diskursiven Schlaufen konfrontieren das traditionelle Vertragsrecht mit schwierigen, zum Teil unlösbaren Problemen:

Denn auf den internen Bildschirmen des Rechtssystems erscheinen diese Reflexionsschlaufen nicht als solche, sondern als etwas, das zunächst in die Rechtssprache »übersetzt « werden muss. Die erfolgte Analyse der jüngeren Rechtsprechung zu den Vertragsverbindungen hat erkennen lassen, dass das Recht die in anderen Sozialsystemen stattfindenden wechselseitigen Reflexionen zwischen Diskursen, die je von verschiedenen Verträgen ausgelöst werden, als Sachverhalt des zusammengesetzten Vertrages rekonstruiert (und eben gerade nicht als verschleifte Reflexionsoperationen - was nur für den Rechtssoziologen, nicht aber für den Juristen etwas bedeutet). Diese Rekonstruktion, die - der systemtheoretischen Grundregel entsprechend - sich ausschließlich an der Leitdifferenz des Rechtssystems orientiert, ist jedoch in dem Sinne defizitär, als sie die (systemrelative) Wirklichkeit der beobachteten Operationen nicht einzufangen ver-

86 Vgl. ausführlich zum Ganzen Gunther Teubner (1997) »Im blinden Fleck der Systeme: Die Hybridisierung des Vertrages«, 3 Soziale Systeme, 313-326; ferner das Beispiel bei dems. (Fn. 82) 249 f.: »Wenn eine medizinische Operation durchgeführt, ein technisches Projekt erstellt, eine komplexe Dienstleistung erbracht werden soll, aktualisiert die vertragliche Beziehung dieses Potential und wandelt es in die verbindliche Struktur eines Sozialsystems, in die Verpflichtung eines Diskurses und in die reale Leistung einer sozialen Dynamik um«.

87 Teubner (Fn. 82) 314. 
mag: Das Recht verliert die Übersicht darüber, welchem der verschiedenen Diskurse, die an einem Vertragsnetz beteiligt sind, ein in diesem Kreis aufgetretener Konflikt zuzuweisen ist. Denn die beteiligten Diskurse beobachten den Konflikt aus der je eigenen Perspektive und führen ihn in das je eigene Entscheidungskalkül ein. Deshalb ist der Konflikt sozusagen in jedem der beteiligten Diskurse »präsent«, was im Rechtssystem Unübersichtlichkeit produziert: Dieses vermag nicht mit der erforderlichen Genauigkeit zu erkennen, ob nun der Konflikt in den Kontext des Vertrages A oder in denjenigen des Vertrages B gehört. Es diagnostiziert dann eine »Vertragsverbindung «, ohne dass aber mit diesem Befund mehr als die Bezeichnung eines Problems gewonnen wäre.

Das lässt sich plastisch am Beispiel des vorne dargestellten BGE 121 III 310 veranschaulichen: Unklar war aus juristischer Perspektive, ob der Konflikt (der von der weisungswidrigen Freigabe des Sperrkontos durch die Bank Y. [Empfängerbank] ausgelöst wurde) in den Kontext des Vertrages zwischen der Bank Z. (Absenderbank) und der Bank Y. oder in denjenigen des Vertrages zwischen dem T. (Hauptauftraggeber) und der Bank Z. gehört. Letztlich muss die Antwort paradox ausfallen: Weder in den einen noch in den anderen und dennoch in beide! Und diesem Paradox entkommt man nur, wenn dieses auf einer »höheren « Ebene, nämlich auf derjenigen des rechtsgeschäftlichen Verbundes, entfaltet wird (was das Bundesgericht denn auch getan hat).

Vermag nun das Seinsbild der Vertragsverbindung, das durch evolutionstheoretische Beobachtungen gewonnen wurde, an dieser Stelle weiterzuhelfen? Entscheidend ist in diesem Zusammenhang die Erkenntnis, dass das Netzwerk der Verträge »constraints« besitzt, die den »Sinn « des Gesamtgebildes auf eine emergente Ebene verlagern - auf die Ebene der durch Symbiose der Verträge gebildeten Ordnung. Entsprechend sollten Konflikte, die in diesem Netzwerk entstehen, auf dieser »höheren« Ebene angegangen werden, nicht auf derjenigen der Einzelverträge, deren jeweilige Einschlägigkeit für diese Konflikte, wie gesagt, aus der Perspektive des Rechts unklar ist. Das Problem liegt aber gerade hierin: das Gesamtgebilde hat de jure keine Ordnung, es besitzt keine Rechtsverfassung, die eine juristische Entscheidung des Konfliktes unter Berücksichtigung der »Netzwerk «-Logik erlauben würde. Damit fragt sich, wie der rechtsgeschäftliche Verbund einer umfassenden Ordnung zugeführt werden kann. Mein Vorschlag geht dahin, dass auf Ebene des Vertragsrechts mit denselben Gesetzmäßigkeiten (Algorithmen!) gearbeitet wird, die in Wirtschaft und Gesellschaft die evolutionäre Herausbildung von Vertragsverbindungen bestimmen:

Dass nämlich die Vertragsverbindung zu einer neuen, emergenten Erwartungsordnung führt, zu einer »höheren« Ordnung, die einer »Netzwerk«-Logik untersteht, indiziert die Vermutung, dass die Regeln, die für die einzelnen Verbundverträge ex lege gelten, das Potential besitzen, für das gesamte Netz »weitergedacht« zu werden. Der Emergenz einer »höheren « Erwartungsordnung entspräche dann die Emergenz einer »höheren« Rechts-Ordnung auf der Basis der für die einzelnen Verbundverträge geltenden Rechts-Regeln. Konkret ginge es darum, aus den »Grundregeln« des klassischen Vertragsrechts eine emergente schuldrechtliche Ordnung für den rechtsgeschäftlichen Verbund herzuleiten. Anstatt von Grund auf eine neue Verbundverfassung zu konstruieren, die bisher dem Obligationenrecht unbekannte dogmatische Elemente verwendet, würden die Normen, die die einzelnen Verbundverträge in das Vertragsnetzwerk »ein- 
bringen «, als Bausteine für eine Rechtsverfassung des Ganzen dienen ${ }^{88}$. Die Frage ist dann nur, nach welchen Gesichtspunkten ein derartiges, netzwerkbezogenes »Weiterdenken« klassischen Vertragsrechts vorzunehmen ist. Ich möchte es mit folgender These versuchen: Die Unterstellung von »Netzwerkkonflikten« unter Regeln, die die einzelnen Verträge in das Netzwerk »einbringen«, bedeutet im Grunde genommen, diesen Regeln eine zweite (emergente) Dimension zu verleihen. Damit sie diese »zweite« Dimension erlangen können, muss ihre Anwendbarkeit nicht an den üblichen, für bipolare Rechtsgeschäfte gedachten Kriterien gemessen werden, sondern von einem neuen Anwendungsmaßstab abhängig gemacht werden. Eine juristische Technik, die geeignet ist, einen solchen Maßstab zur Verfügung zu stellen, ist die kollisionsrechtliche Methode ${ }^{89}$.

Um diese These zu begründen, ist von einem Vergleich der Grundproblematik des Vertragsverbundes mit derjenigen des IPR auszugehen. Zwar liegt bei vernetzten Verträgen nicht ein plurinationaler, sondern ein »plurivertraglicher « Sachverhalt vor; in beiden Fällen hat man es aber letztlich mit demselben rechtstechnischen Problem zu tun: nach welchem »Statut « (im IPR: nach welcher staatlichen Rechtsordnung; im Fall des rechtsgeschäftlichen Verbundes: nach welchem Vertrag im Netz) ist der aus dem Sachverhalt sich ergebende Konflikt zu beurteilen? Für die Vertragsverbindung kann dieses Problem auf die Frage reduziert werden: Wie lässt sich die Zuweisung von im Netzverbund auftretenden Konflikten zu einzelnen Verbundverträgen (zu den dafür geltenden Rechtsregeln) nach »netzlogischen« Kriterien durchführen? Gelänge es, solche Kriterien zu entwickeln, würden die auf einzelvertraglicher Ebene geltenden Normen auf die »höhere « Ebene der Vertragsverbindung »gehoben« und dort - qua kollisionsrechtlicher Verweisung - zu einer Rechtsverfassung des Ganzen gebündelt. Eine Möglichkeit, um solches zu realisieren, besteht darin, sich durch SCHNITZERs Lehre von der funktionellen Anknüpfung inspirieren zu lassen: »[E]ntscheidend für die $\mathrm{Zu}-$ ordnung [eines Rechtsverhältnisses ist] die Funktion dieses Rechtsverhältnisses ..., da ja ein Rechtsverhältnis Rechte und Pflichten zwischen Personen ordnet, die eben funktionell einzuordnen sind ${ }^{90}$. Bezogen auf den rechtsgeschäftlichen Verbund wäre aus dieser Überlegung abzuleiten, dass für die Zuordnung eines Konfliktes zu einem bestimmten Verbundvertrag der Gesichtspunkt, welche Funktion dieser Vertrag im Netzwerk erfüllt, wegleitend sein muss. Oder anders gewendet: Das »sachgerechte« Kriterium zur Artikulierung der gesuchten Kollisionsregel müsste die Funktionsfähigkeit des Vertragsnetzes sein. Entsprechend ließe sich diese Regel dahingehend formulie-

88 Dieser Prozess der Verfassung (in der aktiven Sinnvariante des Begriffes: die verfassende Tätigkeit) der Vertragsverbindung erfolgte in diesem Fall nach einem Muster, das Gunther Teubner (2003) »Globale Zivilverfassungen: Alternativen zur staatszentrierten Verfassungstheorie«, 63 ZaöRV, 1-28, 18, in äußerst greifbarer Weise beschrieben hat: »Verfassung ist immer erst die Verknüpfung zweier realer Prozesse: Aus der Sicht des Rechts ist sie Rechtsnormenproduktion, die eigentümlich mit Grundstrukturen des Sozialsystems verflochten ist; aus der Sicht des verfassten Sozialsystems ist sie Erzeugung von Grundstrukturen der Sozialordnung, die zugleich das Recht informieren und ihrerseits vom Recht normiert werden «.

89 Vgl. hierzu eingehender Amstutz (Fn. 78) $170 \mathrm{ff}$.

90 Adolf Schnitzer (1968) »Die funktionelle Anknüpfung im internationalen Vertragsrecht«, in: Rechts-, wirtschafts- und sozialwissenschaftliche Fakultät der Universität Freiburg (Hg.) Festgabe für Wilhelm Schönenberger, Freiburg i.Ue.: Universitätsverlag, 387-404, 397 (H.v.Verf.). 
ren, dass Konflikte im rechtsgeschäftlichen Verbund demjenigen Vertrag unterstehen, dessen Regeln im konkreten Fall die Funktionsfähigkeit des Vertragsnetzes absichern.

Schimmert eine solche Regel durch die Rechtsprechung des Bundesgerichts durch? Sollte man versuchen, die dogmatisch doch unterbelichteten Konstruktionen in BGE 115 II 452 und 121 III 310 auf dieser Grundlage zu erklären? Kurzum: Sind diese Entscheidungen die Wiege einer neuen »Netzwerk«-Dogmatik im Schuldrecht? Ex facto ius oritur? Eines jedenfalls ist sicher: Im Ergebnis wurde in BGE 115 II 452 die »anzuknüpfende « Rechtsfrage nach der Beendigung des Mietverhältnisses den Regeln des Zusammenarbeitsvertrags zugewiesen. Und in BGE 121 III 310 wurde der Schadenersatzanspruch des T. als vertraglicher qualifiziert, was nur bedeuten kann, dass die entbrannte Streitigkeit nach den Regeln entweder des Hauptauftrages (T. / Bank Z.) oder des Substitutionsauftrages (Bank Z. / Bank Y.) geschlichtet wurde, dass mithin jedenfalls eine kollisionsrechtliche Verweisung stattgefunden hat (auch wenn nach der Lektüre der Urteilserwägungen unklar bleibt, auf welchen Vertrag konkret verwiesen wurde).

Ist man bereit, dieser These zu folgen und Vertragsverbindungen mit Hilfe einer im geschilderten Sinne funktionalen Kollisionsregel zu verfassen, so fragt sich freilich, was unter dem Kriterium der »Funktionsfähigkeit des Vertragsnetzes« konkret zu verstehen ist. Das ist wohl eine Frage, auf die keine generalisierte Antwort möglich ist; vermutlich kennt die Realität eine Unzahl verschiedener Typen von Vertragsverbindungen, die nach unterschiedlichen Modi funktionieren ${ }^{91}$. Eine phänomenologische Studie all dieser Erscheinungsformen ist hier nicht zu leisten. Demgegenüber kann aber nachgewiesen werden, dass das geschriebene Recht bei einzelnen Vertragsverbindungen (insbesondere: Art. 262 Abs. 3 Satz 2, Art. 268 Abs. 2, Art. 291 Abs. 3 Satz 2, Art. 299c, Art. 399 Abs. 3 OR, Art. 21 KKG) netzfunktionale Kriterien verwendet, um Zuordnungsfragen der Art, wie sie vorliegend diskutiert werden, zu lösen, und auf diese Weise Vertragsverbindungen schuldrechtlich verfasst.

Interessant ist diese Feststellung nicht nur deshalb, weil daraus hervorgeht, dass sich die vorliegend entwickelte Lösung zwangslos in die Architektur des schweizerischen Vertragsrechts eingliedern lässt ${ }^{92}$, sondern auch deshalb, weil so, wie noch zu zeigen

91 Vgl. etwa Philipp Hessinger (2001) Vernetzte Wirtschaft und ökonomische Entwicklung: Organisatorischer Wandel, institutionelle Einbettung, zivilgesellschaftliche Perspektive, Wiesbaden: Westdeutscher Verlag, 196 ff.

92 Namentlich steht die hier präkonisierte Lösung nicht im Widerspruch zur Privatautonomie. Diese Frage kann hier freilich nicht vertieft werden. Immerhin soviel: Die Schuljurisprudenz begründet den Grundsatz der gegenseitigen Unabhängigkeit der Verträge im rechtsgeschäftlichen Verbund unter Berufung auf die Relativität von Obligationen und auf die Privatautonomie (nahezu paradigmatisch Cerutti (Fn. 35) 54 ff. N 214 ff.). In einer akribischen Untersuchung über den Subunternehmervertrag hat Chaix (Fn. 46) 91 ff., nachgewiesen, dass dieser Grundsatz in zahlreichen Fällen zu unbefriedigenden Lösungen führt, die für ein modernes Privatrecht nicht akzeptabel sind. Folgt man ferner der hier vertretenen These, dass die Differenzierung der Gesellschaft das Vertragsrecht mit Netzkonstruktionen konfrontiert, für die die klassischen schuldrechtlichen Lösungsmuster nicht mehr ausreichen, muss dieses Recht neue Antworten entwickeln. Denn diese Netzkonstruktionen entsprechen realen Bedürfnissen in der Lebenswelt, denen das Rechtssystem gerecht werden muss (vgl. statt aller Walter Schluep (1979) »Innominatverträge«, in: Frank Vischer (Hg.) Schweizerisches Privatrecht: Bd. VII/2: Obligationenrecht: Besondere Vertragsverhältnisse, Basel: Helbing \& Lichtenhahn, 798). 
ist, erste Funktionstypen von Vertragsverbindungen ins Blickfeld geraten. Selbstredend kann an dieser Stelle nicht auf sämtliche eben erwähnten Normen des positiven Rechts eingegangen werden. Aufgegriffen (und auch dies nur skizzenhaft) werden lediglich Art. 262 Abs. 3 Satz 2 sowie Art. 399 Abs. 3 OR, die zwei unterschiedliche, auf den geschilderten kollisionrechtlichen Mechanismus gestützte Verbundverfassungstechniken zu illustrieren erlauben. Nach Art. 262 Abs. 3 Satz 2 OR kann bekanntlich der Hauptvermieter nicht nur den Untervermieter (d.h. seinen eigenen Mieter), sondern auch den Untermieter (mit dem ihn kein Vertragsverhältnis verbindet) unmittelbar zu einem Gebrauch der Mietsache, der dem Hauptmietvertrag entspricht, anhalten $^{93}$. Nach Art. 399 Abs. 3 OR kann sodann beim Mandat in Fällen der (sowohl befugten als auch unbefugten) Substitution der Auftraggeber die Ansprüche, die dem Beauftragten gegen den Substituten zustehen, selber direkt gegen diesen (mit dem er wohlbemerkt keine Vereinbarung abgeschlossen hat) geltend machen ${ }^{94}$. CERUTTI hebt hervor, dass diese Normen die gemeinsame Besonderheit aufweisen, »dass sie dem Erstkontrahenten [sc. dem ersten Glied in der von Haupt- und Untervertrag gebildeten Vertragskette] nur gerade die vom Gesetzgeber für den jeweiligen Untervertrag als notwendig erachteten Begünstigungen einräumen $«{ }^{95}$. Das wirft die Frage auf, was dieses »Notwendige« ist bzw. welches das entscheidende Kriterium ist, um im Vertragsnetz Rechts- und Pflichtenpositionen zu allozieren.

Betrachtet man zunächst Art. 262 Abs. 3 Satz 2 OR genauer, fällt auf, dass dem Vermieter zwar gestattet wird, den Untermieter direkt zur Einhaltung der Verwendungszwecksklauseln im Hauptmietvertrag aufzufordern, dass ihm aber das Recht verweigert bleibt, von ihm Leistung des (Unter-) Mietzinses zu verlangen ${ }^{96}$. Zieht man die mit jedem Überlassungsvertrag einhergehende Obhutsproblematik in Betracht ${ }^{97}$, wird das netzfunktionale Motiv dieser Regel offenkundig: Der mietrechtliche Verbund (als Netzwerk von interessengegensatzbezogenen Kontrakten) kann schon von seiner Anlage her keine Vergemeinschaftung der beteiligten finanziellen Interessen zur Folge haben; seine Funktionsfähigkeit hängt aber von der Überlassungstauglichkeit der Mietsache ab, die (im Einklang mit dem stets noch vorherrschenden Grundprinzip der Marktgesellschaft) von demjenigen festgelegt wird, der das Eigentum daran innehat, also vom (Haupt-) Vermieter. Dementsprechend dient Art. 262 Abs. 3 Satz 3 OR dazu, den Ansporn zur Substanzerhaltung des Überlassungsobjekts netzweit zu verteilen, indem der »Motor« dieses Ansporns - das Eigentum - in sämtlichen Verästelungen des Verbundes »wirken« kann.

93 Vgl. Higi (Fn. 43) 664 N 27 m.w.N.; David Lachat (2003) »Art. 262«, in: Luc Thévenoz und Franz Werro (Hg.) Commentaire Romand: Code des obligations I (Code des obligations art. 1-529; Loi sur le crédit à la consommation, Loi sur les voyages à forfait), Genève: Helbing \& Lichtenhahn, 1371 N 7.

94 Vgl. generell Walter Fellmann (1992) »Art. 394-406 OR«, in: Heinz Hausheer (Hg.) Berner Kommentar: Das Obligationenrecht, Bd. VI / 2. Abt. / 4. Teilbd.: Der einfache Auftrag, Bern: Stämpfli, 537 ff. N 610 ff., 577 ff. N 92 ff. m.w.N.

95 Cerutti (Fn. 35) 39 N 149.

96 Cerutti (Fn. 35) 39 N 150; Chaix (Fn. 46) 205 f. m.w.N.

97 Zur Obhutsproblematik Josef Esser und Hans-Leo Weyers (1998) Schuldrecht, Bd. II: Besonderer Teil, Teilbd. 1: Verträge, 8. Aufl., Heidelberg: C.F. Müller, 130. 
Eine andere »Netzwerk«-Logik durchzieht Art. 399 Abs. 3 OR $^{98}$ : Der Auftrag zeichnet sich im Hinblick auf das Erfüllungsprogramm des Mandataren durch eine hohe Unbestimmtheit aus ${ }^{99}$. Diese ist Korrelat des Umstandes, dass der Beauftragte regelmässig ein hochspezialisierter Fachmann (Patentanwalt, Herzchirurg usw.) ist, weshalb es dem Auftraggeber mangels einschlägigen Wissens faktisch oft unmöglich ist, dieser Person inhaltliche Weisungen zu erteilen. Überbrückt wird dieses Konkretisierungsdefizit durch die Ausrichtung der Vertragserfüllungspflichten auf die Interessen des Mandanten (Grundsatz der Zweckgerichtetheit des Auftrags in Wahrung fremder Interessen; »obligation de moyens«). In Substitutionsverhältnissen wird die geschilderte Unbestimmtheit in dem Ausmaß verschärft, als das Erfüllungsprogramm des Substituten nicht unmittelbar an die Interessen des Auftraggebers zurückgebunden ist $^{100}$. Dem hilft Art. 399 Abs. 3 OR dadurch ab, dass er einen netzweiten Rückkoppelungsmechanismus installiert: ${ }^{101}$ Indem dem Mandanten ein direkter Zugriff auf den Substituten zwecks Durchsetzung der vom Substitutionsauftrag (also dem »Untervertrag «) geschaffenen Pflichtenlage gewährt wird, werden im Netz der nachgeschalteten Aufträge die »incentives « für die Beteiligten so plaziert, dass das Erfüllungsprogramm des Substituten nach Möglichkeit auf die Interessen des Mandataren ausgerichtet wird $^{102}$.

Die Aufdeckung des »netzfunktionalen« Kalküls, auf dem Art. 262 Abs. 3 Satz 2 und Art. 399 Abs. 3 OR gründen, eröffnet die Möglichkeit, diese Bestimmungen als »schuldrechtliche Netz-Verfassungsnormen « zu verstehen. Aus den vorgetragenen Überlegungen geht insbesondere hervor, dass sowohl Art. 262 Abs. 3 Satz 2 als auch Art. 399 Abs. 3 OR auf dem Gesichtspunkt der Funktionsfähigkeit des Vertragsverbundes aufbauen, dass hingegen die Konkretisierung dieses Merkmales je nach spezifischem Funktionsmodus des in Frage stehenden »Netzes « verschiedenartig geschieht: Während es bei Art. 262 Abs. 3 Satz 2 OR um die Ausdehnung der im Hauptmietvertrag »angesiedelten« Eigentümerstellung des Hauptvermieters im mietgeschäftlichen

98 Wie Chaix (Fn. 46) 234 ff., dartut, ist die Frage nach dem Zweck von Art. 399 Abs. 3 OR im schweizerischen Schrifttum noch unterbelichtet; vgl. zu dieser Frage vor allem Fellmann (Fn. 94) 577 N 93; Cerutti (Fn. 35) 45 N 179; Peter Derendinger (1988) Die Nichtund die nicht richtige Erfüllung des einfachen Auftrages, Freiburg i.Ue.: Universitätsverlag, 146 f. N 324; Josef Hofstetter (2000) »Der Auftrag und die Geschäftsführung ohne Auftrag «, in: Wolfgang Wiegand (Hg.) Schweizerisches Privatrecht: Bd. VII/6: Obligationenrecht - Besondere Vertragsverhältnisse, Basel: Helbing \& Lichtenhahn, 98.

99 Vgl. statt aller Franz Werro (1993) Le mandat et ses effets: Une étude sur le contrat d'activité indépendante selon le Code suisse des obligations: Analyse critique et comparative, Freiburg i.Ue.: Universitätsverlag, 192 f. N 560 ff.

100 Zutreffend Werro (Fn. 99) 191 N 558.

101 In diese Richtung argumentiert wohl auch Fellmann (Fn. 94) 540 f. N 618 f.

102 In diesem Sinne wohl auch Chaix (Fn. 46) 237; deutlich erkennbar wird diese Zweckausrichtung an der (zutreffenden) Ansicht des Bundesgerichts in BGE 110 II $186 \mathrm{f}$., dass die Ansprüche nach Art. 399 Abs. 3 OR auch das Widerrufsrecht nach Art. 404 OR umfassen; vgl. Chaix a.a.O., 209 m.w.N.; Tercier (Fn. 72) 672 N 4661; zu Unrecht ablehnend (mit obendrein fragwürdigen Argumenten) Fellmann (Fn. 94) 580 N 103; ernster zu nehmen sind demgegenüber die ebenfalls ablehnenden Argumente von Cerutti (Fn. 35) 40 N 154, 119 f. N 477 ff. 
Verbund geht, bezweckt Art. 399 Abs. 3 OR die Rückbindung des im Unterauftrag »angesiedelten « Erfüllungsprogramms des Substituten an die Interessen des Mandanten. Entsprechend unterschiedlich gestalten sich dann auch die jeweiligen Verfassungen des mietvertraglichen bzw. des auftragsrechtlichen Verbundes: Art. 262 Abs. 3 Satz 2 OR unterstellt die Schuldnerstellung (die Stellung des Untermieters) einem Vertrag (dem Hauptmietvetrag), in dem der Schuldner formell keine Parteistellung hat. Demgegenüber unterstellt Art. 399 Abs. 3 OR die Gläubigerstellung (die Stellung des Auftraggebers) einem Vertrag (dem Substitutionsauftrag), in dem der Gläubiger formell keine Parteistellung hat ${ }^{103}$. Damit wird der Rechtsanwender in die Lage versetzt, über die spezifische Konkretisierung des Gesichtspunktes der netzbezogenen Funktionsfähigkeit die jeweils zu beurteilende Rechtsfrage (bei Art. 262 Abs. 3 Satz 2 OR: die Nutzung der Untermietsache; bei Art. 399 Abs. 3 OR: die Durchsetzung des Erfüllungsprogramms des Substituten) an die »sachgerechte« Ordnung (bei Art. 262 Abs. 3 Satz 2 OR: an den Obervertrag; bei Art. 399 Abs. 3 OR: an den Untervertrag) anzuknüpfen und dadurch eine adäquate schuldrechtliche Verfassung zu gestalten.

103 Diesen ganz unterschiedlichen Strukturen von Art. 262 Abs. 3 Satz 2 und Art. 399 Abs. 3 OR wird Fellmann (Fn. 94) 578 N 97, nicht gerecht, wenn er davon spricht, dass in beiden Fällen ein »einseitig berechtigendes Schuldverhältnis« geschaffen wird. Demgegenüber differenziert Chaix (Fn. 46) 185 ff., ähnlich wie hier, zwischen »effets négatifs du contrat« und »effets positifs du contrat «. 